FERMILAB-Pub-95/031

$\mathrm{MAD} / \mathrm{PH} / 871$

UB-HET-95-01

UdeM-GPP-TH-95-14

March 1995

\title{
ANOMALOUS GAUGE BOSON INTERACTIONS O
}

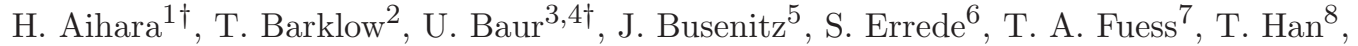 \\ D. London ${ }^{9 \dagger}$, J. Ohnemus ${ }^{8}$, R. Szalapski ${ }^{10}$, C. Wendt ${ }^{11}$, and D. Zeppenfeld ${ }^{11 \dagger}$ \\ ${ }^{1}$ Lawrence Berkeley Laboratory, Berkeley, CA 94720 \\ ${ }^{2}$ Stanford Linear Accelerator Center, Stanford, CA 94309 \\ ${ }^{3}$ Physics Department, SUNY Buffalo, Buffalo, NY 14260 \\ ${ }^{4}$ Physics Department, Florida State University, Tallahassee, FL 32306 \\ ${ }^{5}$ Department of Physics and Astronomy, University of Alabama, Tuscaloosa, AL 35487 \\ ${ }^{6}$ Physics Department, University of Illinois at Urbana - Champaign, Urbana, IL 61801 \\ ${ }^{7}$ Argonne National Laboratory, Argonne, IL 60439 \\ ${ }^{8}$ Physics Department, University of California, Davis, CA 95616 \\ ${ }^{9}$ Physics Department, University of Montreal, Canada H3C $3 J^{7}$ \\ ${ }^{10}$ Theory Group, KEK, Tsukuba, Ibaraki 305, Japan \\ ${ }^{11}$ Physics Department, University of Wisconsin, Madison, WI 53706
}

\begin{abstract}
We discuss the direct measurement of the trilinear vector boson couplings in present and future collider experiments. The major goals of such experiments will be the confirmation of the Standard Model (SM) predictions and the search for signals of new physics. We review our current theoretical understanding of anomalous trilinear gauge-boson self interactions. If the energy scale of the new physics is $\sim 1 \mathrm{TeV}$, these low energy anomalous couplings are expected to be no larger than $\mathcal{O}\left(10^{-2}\right)$. Constraints from high precision measurements at LEP and low energy charged and neutral current processes are critically reviewed.
\end{abstract}

\section{Introduction}

Over the last five years $e^{+} e^{-}$collision experiments at LEP and at the SLAC linear collider have beautifully confirmed the predictions of the Standard Model $(\mathrm{SM})$. At present experiment and theory agree at the $0.1-1 \%$ level in the determination of the vector boson couplings to the various fermions [1], which may rightly be considered a confirmation of the gauge boson nature of the $W$ and the $Z$. Nevertheless the most direct consequences of the $S U(2)_{L} \times U(1)_{Y}$ gauge symmetry,

\footnotetext{
${ }^{*}$ Summary of the Working Subgroup on Anomalous Gauge Boson Interactions of the DPF Long-Range Planning Study, to be published in "Electroweak Symmetry Breaking and Beyond the Standard Model", eds. T. Barklow, S. Dawson, H. Haber and J. Siegrist.

${ }^{\dagger}$ Co-convener
} 
the non-abelian self-couplings of the $W, Z$, and photon, remain poorly measured to date.

A direct measurement of these vector boson couplings is possible in present and future collider experiments, in particular via pair production processes like $e^{+} e^{-} \rightarrow W^{+} W^{-}, Z \gamma$ and $q \bar{q} \rightarrow W^{+} W^{-}, W \gamma, Z \gamma, W Z$. The first and major goal of such experiments will be a confirmation of the SM predictions. A precise and direct measurement of the trilinear and quartic couplings of the electroweak vector bosons and the demonstration that they agree with the SM would beautifully corroborate spontaneously broken, non-abelian gauge theories as the basic theoretical structure describing the fundamental interactions of nature.

At the same time, such measurements may be used to probe for new physics. Since the gauge boson self-couplings have not yet been measured with good precision, it is possible in principle that signals for physics beyond the SM will appear in this sector through the discovery of anomalous trilinear (or quartic) gauge-boson vertices (TGV's). This possibility immediately raises a number of other questions. What are the expected sizes of such anomalous effects in different models of new physics? Will the new physics which gives rise to anomalous gauge-boson couplings manifest itself in other observables and/or other channels? Are there significant constraints from low-energy measurements? We address these questions in Section 2.

For the most part, however, we are interested in the accuracy of various collider experiments for a direct measurement of the self-interactions of electroweak vector bosons, so as to evaluate how well the SM predictions can be tested (Section 3). For simplicity, we shall restrict ourselves to trilinear vector boson couplings, in particular the $W W V$, and $Z \gamma V, V=\gamma, Z$ couplings. Possibilities to test quartic couplings in collider experiments are discussed in Ref. [2].

Analogous to the introduction of arbitrary vector and axial vector couplings $g_{V}$ and $g_{A}$ for the coupling of gauge bosons to fermions, the measurement of the $W W V$ couplings can be made quantitative by introducing a more general $W W V$ vertex. For our discussion of experimental sensitivities in Section 3 we shall use a parameterization in terms of the phenomenological effective Lagrangian [3]

$$
\begin{aligned}
i \mathcal{L}_{e f f}^{W W V}= & g_{W W V}\left[g_{1}^{V}\left(W_{\mu \nu}^{\dagger} W^{\mu}-W^{\dagger \mu} W_{\mu \nu}\right) V^{\nu}+\kappa_{V} W_{\mu}^{\dagger} W_{\nu} V^{\mu \nu}+\right. \\
& \left.\frac{\lambda_{V}}{m_{W}^{2}} W_{\rho \mu}^{\dagger} W^{\mu}{ }_{\nu} V^{\nu \rho}+i g_{5}^{V} \varepsilon_{\mu \nu \rho \sigma}\left(\left(\partial^{\rho} W^{\dagger \mu}\right) W^{\nu}-W^{\dagger \mu}\left(\partial^{\rho} W^{\nu}\right)\right) V^{\sigma}\right] .
\end{aligned}
$$

Here the overall couplings are defined as $g_{W W \gamma}=e$ and $g_{W W Z}=e \cot \theta_{W}, W_{\mu \nu}=$ $\partial_{\mu} W_{\nu}-\partial_{\nu} W_{\mu}$, and $V_{\mu \nu}=\partial_{\mu} V_{\nu}-\partial_{\nu} V_{\mu}$. Within the SM, at tree level, the couplings are given by $g_{1}^{Z}=g_{1}^{\gamma}=\kappa_{Z}=\kappa_{\gamma}=1, \lambda_{Z}=\lambda_{\gamma}=g_{5}^{Z}=g_{5}^{\gamma}=0$. For on-shell photons, $g_{1}^{\gamma}=1$ and $g_{5}^{\gamma}=0$ are fixed by electromagnetic gauge invariance; $g_{1}^{Z}$ and $g_{5}^{Z}$ may, however, differ from their SM values. Deviations are given by the anomalous TGV's

$$
\Delta g_{1}^{Z} \equiv\left(g_{1}^{Z}-1\right), \quad \Delta \kappa_{\gamma} \equiv\left(\kappa_{\gamma}-1\right), \quad \Delta \kappa_{Z} \equiv\left(\kappa_{Z}-1\right), \quad \lambda_{\gamma}, \quad \lambda_{Z}, \quad g_{5}^{Z} .
$$

As we discuss in Section 2, theoretical arguments suggest that these anomalous TGV's are at most of $\mathcal{O}\left(m_{W}^{2} / \Lambda^{2}\right)$, where $\Lambda$ is the scale of new physics (some are 
expected to be considerably smaller). Thus, for $\Lambda \sim 1 \mathrm{TeV}$, the anomalous TGV's are $\mathcal{O}\left(10^{-2}\right)$, which will make their observation difficult. Conversely, if large anomalous TGV's are discovered, this implies that the new physics responsible for them is likely to be found directly below the TeV scale.

The effective Lagrangian of Eq. (11) parameterizes the most general Lorentz invariant and $C P$ conserving $W W V$ vertex which can be observed in processes where the vector bosons couple to effectively massless fermions. Apart from $g_{5}^{V}$, all couplings conserve $C$ and $P$ separately. If $C P$ violating couplings are allowed, three additional couplings, $g_{4}^{V}, \tilde{\kappa}_{V}$ and $\tilde{\lambda}_{V}$, appear in the effective Lagrangian [3] and they all vanish in the SM, at tree level. For simplicity, these couplings are not considered in this report. Terms with higher derivatives are equivalent to a dependence of the couplings on the vector boson momenta and thus merely lead to a form-factor behaviour of these couplings (see Section 2.3). The $C$ and $P$ conserving terms in $\mathcal{L}_{e f f}^{W W \gamma}$ correspond to the lowest order terms in a multipole expansion of the $W$-photon interactions, the charge $Q_{W}$, the magnetic dipole moment $\mu_{W}$ and the electric quadrupole moment $q_{W}$ of the $W^{+}$《田:

$$
\begin{aligned}
Q_{W} & =e g_{1}^{\gamma}, \\
\mu_{W} & =\frac{e}{2 m_{W}}\left(g_{1}^{\gamma}+\kappa_{\gamma}+\lambda_{\gamma}\right), \\
q_{W} & =-\frac{e}{m_{W}^{2}}\left(\kappa_{\gamma}-\lambda_{\gamma}\right) .
\end{aligned}
$$

Analogous to the general $W W V$ vertex it is possible to parameterize anomalous $Z \gamma V, V=\gamma, Z$ couplings. We shall be interested in constraints from $Z \gamma$ production processes in Section 3, i.e. we may treat the photon and the $Z$ as being on-shell. As before we are only considering $C P$-even couplings. Let us denote the Feynman rule for the $V_{\mu}(P) \rightarrow Z_{\alpha}\left(q_{1}\right) \gamma_{\beta}\left(q_{2}\right)$ vertex by $i e \Gamma_{Z \gamma V}^{\alpha \beta \mu}\left(q_{1}, q_{2}, P\right)$. The most general such vertex compatible with Lorentz invariance has been discussed in Ref. [3] and it can be parameterized in terms of two free parameters, $h_{3}^{V}$ and $h_{4}^{V}$,

$$
\Gamma_{Z \gamma V}^{\alpha \beta \mu}\left(q_{1}, q_{2}, P\right)=\frac{P^{2}-m_{V}^{2}}{m_{Z}^{2}}\left[h_{3}^{V} \varepsilon^{\mu \alpha \beta \rho} q_{2 \rho}+\frac{h_{4}^{V}}{m_{Z}^{2}} P^{\alpha} \varepsilon^{\mu \beta \rho \sigma} P_{\rho} q_{2 \sigma}\right] .
$$

Within the SM, at tree level, $h_{3}^{V}=h_{4}^{V}=0$. If $C P$ violating couplings are allowed, two additional couplings, $h_{1}^{V}$ and $h_{2}^{V}$, appear in the effective Lagrangian [3] which also vanish in the SM, at tree level. For simplicity, these couplings are not considered here. The overall factor $P^{2}-m_{V}^{2}$ in Eq. (6) is implied by Bose symmetry for onshell $V$ and/or by gauge invariance for $V=\gamma$. These additional factors indicate that anomalous $Z \gamma V$ couplings can only arise from higher dimensional operators than the $W W V$ couplings and hence their effects should be suppressed in any scenario of new physics beyond the SM.

In Section 3 present measurements from $p \bar{p}$ and $e^{+} e^{-}$collider experiments are summarized. In addition the sensitivity of future Tevatron, LHC, LEP II and 
NLC experiments is analysed in detail. Our conclusions are presented in Section 4.

\section{Theoretical Background}

\subsection{Effective Lagrangians: General Considerations}

In this Section we discuss theoretical ideas which lead to anomalous gauge boson self-interactions, and analyze constraints from low energy and high precision measurements. In the absence of a specific model of new physics, effective Lagrangian techniques are extremely useful. An effective Lagrangian [5] parameterizes, in a model-independent way, the low-energy effects of the new physics to be found at higher energies. It is only necessary to specify the particle content and the symmetries of the low-energy theory. Although effective Lagrangians contain an infinite number of terms, they are organized in powers of $1 / \Lambda$, where $\Lambda$ is the scale of new physics. Thus, at energies which are much smaller than $\Lambda$, only the first few terms of the effective Lagrangian are important.

The Fermi theory of the weak interactions is perhaps the best-known example of an effective Lagrangian. Within the SM, the charged-current interaction between two fermions is described by the exchange of a $W$-boson:

$$
\frac{g^{2}}{8} \bar{\Psi} \gamma_{\mu}\left(1-\gamma_{5}\right) \Psi \frac{1}{q^{2}-m_{W}^{2}} \bar{\Psi} \gamma^{\mu}\left(1-\gamma_{5}\right) \Psi,
$$

where $q^{2}$ is the momentum transfer (energy scale) of the interaction. We can expand the $W$-propagator in powers of $q^{2} / m_{W}^{2}$ :

$$
\frac{1}{q^{2}-m_{W}^{2}}=-\frac{1}{m_{W}^{2}}\left[1+\frac{q^{2}}{m_{W}^{2}}+\ldots\right] .
$$

The interaction of Eq. (7) can thus be written as the sum of an infinite number of terms. However, we note that, for energies well below the $W$ mass, only the first term is important. This is simply the 4-fermion interaction of the Fermi theory:

$$
-\frac{G_{F}}{\sqrt{2}} \bar{\Psi} \gamma_{\mu}\left(1-\gamma_{5}\right) \Psi \bar{\Psi} \gamma^{\mu}\left(1-\gamma_{5}\right) \Psi,
$$

where $G_{F} / \sqrt{2}=g^{2} / 8 m_{W}^{2}$. In other words, the Fermi theory is the effective theory produced when one "integrates out" the heavy degrees of freedom (in this case, the $W$ boson). It is valid at energy scales much less than the scale of heavy physics $\left(q^{2} \ll m_{W}^{2}\right)$.

Note that, as $q^{2}$ approaches $m_{W}^{2}$, one can no longer truncate after the lowestorder term in $q^{2} / m_{W}^{2}$. This is evidence that the effective Lagrangian is breaking down - each of the infinite number of terms becomes equally important as one is approaching energy scales where the heavy degrees of freedom can be directly produced, i.e. they cannot be integrated out. Note also that the truncated effective Lagrangian (the Fermi theory) violates $S$-matrix unitarity for $q^{2}>m_{W}^{2} /\left(g^{2} / 4 \pi\right)$. Unitarity is restored in the full theory by propagator (form factor) effects and the 
scale at which unitarity is apparently violated gives an upper bound for the masses of the heavy degrees of freedom (here the $W$ mass). In a weakly coupled theory like the SM this upper bound substantially overestimates the masses of the heavy degrees of freedom. Apart from resonance enhancement one needs strong interaction dynamics to obtain cross sections in the full theory which approach the unitarity limits. As the energy scale is increased, new channels will open up in addition (e.g. $W W$ and $W Z$ production in the case of the Fermi theory). However, the cross sections of these new channels may be too low to be observable, especially if the underlying dynamics is perturbative in nature. These features, which are easily understood in the context of the SM and the Fermi theory, are general properties of all effective Lagrangians.

\subsection{Power Counting}

In order to define an effective Lagrangian, it is necessary to specify the symmetry and the particle content of the low-energy theory. Since all experimental evidence is consistent with the existence of an $S U(2)_{L} \times U(1)_{Y}$ gauge symmetry it is natural to require the effective Lagrangian describing anomalous TGV's to possess this invariance. Inspecting Eq. (11), the phenomenological effective Lagrangian $\mathcal{L}_{\text {eff }}^{W W V}$ describing anomalous $W W V$ couplings appears not to respect this constraint. This impression is wrong, however, since Eq. (11) can be interpreted as the unitarygauge expression of an effective Lagrangian in which the $S U(2)_{L} \times U(1)_{Y}$ gauge symmetry is manifest [6]. How this symmetry is realized depends on the particle content of the effective Lagrangian. If one includes a Higgs boson, the symmetry can be realized linearly, otherwise a nonlinear realization of the gauge symmetry is required. We will discuss each of these options in turn.

\section{- Linear Realization}

We first consider the linear realization scenario, in which a Higgs doublet field $\Phi$ is included in the low-energy particle content. This is also called the "decoupling physics" scenario in the literature because, with the inclusion of a light Higgs boson, the scale of new physics is allowed to be arbitrarily large, even $\Lambda \sim 10^{15} \mathrm{GeV}$ would be self-consistent. In addition to the Higgs field the building blocks of the effective Lagrangian are covariant derivatives of the Higgs field, $D_{\mu} \Phi$, and the field strength tensors $W_{\mu \nu}$ and $B_{\mu \nu}$ of the $W\left(S U(2)_{L}\right)$ and the $B\left(U(1)_{Y}\right)$ gauge fields:

$$
\left[D_{\mu}, D_{\nu}\right]=\hat{B}_{\mu \nu}+\hat{W}_{\mu \nu}=i \frac{g^{\prime}}{2} B_{\mu \nu}+i g \frac{\sigma^{a}}{2} W_{\mu \nu}^{a} .
$$

Here, $\sigma^{a}, a=1, \ldots, 3$ denote the Pauli matrices, and $g$ and $g^{\prime}$ are the $S U(2)_{L}$ and $U(1)_{Y}$ gauge coupling constants, respectively. Considering dimension-6 operators only, 11 independent such operators can be constructed $[7,8]$ of which only 7 are relevant for our discussion:

$$
\mathcal{L}_{e f f}=\sum_{i=1}^{7} \frac{f_{i}}{\Lambda^{2}} \mathcal{O}_{i}=\frac{1}{\Lambda^{2}}\left(f_{\Phi, 1}\left(D_{\mu} \Phi\right)^{\dagger} \Phi \Phi^{\dagger}\left(D^{\mu} \Phi\right)+f_{B W} \Phi^{\dagger} \hat{B}_{\mu \nu} \hat{W}^{\mu \nu} \Phi\right.
$$




$$
\begin{aligned}
& +f_{D W} \operatorname{Tr}\left(\left[D_{\mu}, \hat{W}_{\nu \rho}\right]\left[D^{\mu}, \hat{W}^{\nu \rho}\right]\right)-f_{D B} \frac{g^{\prime 2}}{2}\left(\partial_{\mu} B_{\nu \rho}\right)\left(\partial^{\mu} B^{\nu \rho}\right) \\
& +f_{B}\left(D_{\mu} \Phi\right)^{\dagger} \hat{B}^{\mu \nu}\left(D_{\nu} \Phi\right)+f_{W}\left(D_{\mu} \Phi\right)^{\dagger} \hat{W}^{\mu \nu}\left(D_{\nu} \Phi\right) \\
& \left.+f_{W W W} \operatorname{Tr}\left[\hat{W}_{\mu \nu} \hat{W}^{\nu \rho} \hat{W}_{\rho}{ }^{\mu}\right]\right) .
\end{aligned}
$$

The first four operators, $\mathcal{O}_{\Phi, 1}, \mathcal{O}_{B W}, \mathcal{O}_{D W}$, and $\mathcal{O}_{D B}$, affect the gauge boson two-point functions at tree level [9] and as a result the coefficients of these four operators are severely constrained by present low energy data. The remaining three, $\mathcal{O}_{B}, \mathcal{O}_{W}$ and $\mathcal{O}_{W W W}$, give rise to non-standard triple gauge boson couplings. Their presence in the effective Lagrangian leads to deviations of the $W W V$ couplings from the SM, namely [8, 10]

$$
\begin{aligned}
& \Delta \kappa_{\gamma}=\left(f_{B}+f_{W}\right) \frac{m_{W}^{2}}{2 \Lambda^{2}}, \Delta \kappa_{Z}=\left(f_{W}-s^{2}\left(f_{B}+f_{W}\right)\right) \frac{m_{Z}^{2}}{2 \Lambda^{2}} \\
& \Delta g_{1}^{Z}=f_{W} \frac{m_{Z}^{2}}{2 \Lambda^{2}}=\Delta \kappa_{Z}+\frac{s^{2}}{c^{2}} \Delta \kappa_{\gamma}, \\
& \lambda_{\gamma}=\lambda_{Z}=\lambda=\frac{3 m_{W}^{2} g^{2}}{2 \Lambda^{2}} f_{W W W},
\end{aligned}
$$

with $s=\sin \theta_{W}$ and $c=\cos \theta_{W}$. Note that all anomalous TGV's are suppressed by a factor $m_{W}^{2} / \Lambda^{2}$ and hence they vanish in the decoupling limit. In fact this behaviour is required by unitarity considerations with, typically, $\left|f_{i}\right| \lesssim 32 \pi$ [11]. In general, the coefficients $f_{i}$ are expected to be numbers of order unity. Hence, taking $\Lambda \sim 1 \mathrm{TeV}$, one might expect anomalous TGV's of $\mathcal{O}\left(10^{-2}\right)$. As pointed out by Einhorn and collaborators [12], the dimension six operators $\mathcal{O}_{W W W}, \mathcal{O}_{W}$ and $\mathcal{O}_{B}$ which lead to anomalous TGV's cannot be generated at the tree level by any renormalizable underlying theory which leads to the effective Lagrangian of Eq. (11). Thus, in this scenario, the expected size of the anomalous TGV's would be tiny, $\sim 1 /(16 \pi)^{2}\left(m_{W}^{2} / \Lambda^{2}\right)$, and only small scales $\Lambda$ would be accessible experimentally. In the same scenario dimension 8 operators leading to TGV contributions can be generated at tree level and, thus, they might dominate over the dimension 6 terms considered above if $\Lambda$ is sufficiently small. Since the correlations between different anomalous $W W V$ couplings exhibited in Eqs. (13) and (14) are due to the truncation of the effective Lagrangian at the dimension six level [8] these relationships would not even be approximately correct in this case.

Anomalous $Z \gamma V$ couplings originate only from terms of dimension 8 or higher in the effective Lagrangian and, therefore, are expected to be $\mathcal{O}\left(m_{Z}^{4} / \Lambda^{4}\right)$.

- Nonlinear Realization

Let us now turn to the scenario in which the $S U(2)_{L} \times U(1)_{Y}$ gauge symmetry is realized non-linearly ("non-decoupling physics"). In this case, one includes only the would-be Goldstone bosons (WBGB's) which give masses to the $W$ - and $Z$ bosons. Since there is no Higgs boson, the low-energy Lagrangian violates unitarity at a scale of roughly $4 \pi v \sim 3 \mathrm{TeV}$, so that the new physics must appear at a scale $\Lambda \lesssim 4 \pi v$. 
A number of nonlinear realizations appear in the literature, all of which are similar [13]. For the purpose of illustration we will choose one which conserves the custodial $S U(2)_{C}$ symmetry of the SM in the limit $g^{\prime} \rightarrow 0$. Using the matrix $\Sigma \equiv \exp (i \vec{\omega} \cdot \vec{\sigma} / v)$, where the $\omega_{i}$ are the WBGB's, we define the $S U(2)_{L} \times U(1)_{Y}$ covariant derivative:

$$
D_{\mu} \Sigma \equiv \partial_{\mu} \Sigma+\frac{i}{2} g W_{\mu}^{a} \sigma^{a} \Sigma-\frac{i}{2} g^{\prime} B_{\mu} \Sigma \sigma_{3} .
$$

One then constructs terms in the effective Lagrangian using field strengths $\left(W_{\mu \nu}\right.$, $\left.B_{\mu \nu}\right)$ and covariant derivatives. This effective Lagrangian is known generically as a "chiral Lagrangian," due to its similarity to low-energy QCD (and chiral perturbation theory). In the unitary gauge the covariant derivative becomes a linear combination of gauge bosons. Thus, a gauge-boson field can be constructed by taking the trace of $D_{\mu} \Sigma$ with the appropriate $\sigma$ matrix, e.g. $Z_{\mu} \sim \operatorname{Tr}\left[\sigma_{3} \Sigma^{\dagger} D_{\mu} \Sigma\right]$. In this way, we can write the Lagrangian of Eq. (11) in terms of $S U(2)_{L} \times U(1)_{Y}$-invariant quantities.

Our experience with QCD tells us how to estimate the size of any term in a chiral Lagrangian. This estimate is called "naive dimensional analysis" (NDA) [14]. It states that a term having $b$ WBGB fields, $f$ (weakly-interacting) fermion fields, $d$ derivatives and $w$ gauge fields has a coefficient whose size is

$$
c_{n}(\Lambda) \sim v^{2} \Lambda^{2}\left(\frac{1}{v}\right)^{b}\left(\frac{1}{\Lambda^{3 / 2}}\right)^{f}\left(\frac{1}{\Lambda}\right)^{d}\left(\frac{g}{\Lambda}\right)^{w} .
$$

Applying NDA to the terms in Eqs. (11) and (2), we see that $\Delta g_{1}^{V}$ and $\Delta \kappa_{V}$ are of $\mathcal{O}\left(m_{W}^{2} / \Lambda^{2}\right)$. In other words, just as in the linear realization, these terms are effectively of dimension 6 (in the sense that there is an explicit factor of $1 / \Lambda^{2}$ ). On the other hand, we see that the $W_{\rho \mu}^{\dagger} W^{\mu}{ }_{\nu} V^{\nu \rho}$ term is effectively of dimension 8, i.e. the coefficient $\lambda_{V}$ is expected to be of order $m_{W}^{4} / \Lambda^{4}$. Thus, within the nonlinear realization scenario, the $\lambda_{V}$ terms are expected to be negligible compared to those proportional to $\Delta g_{1}^{V}$ and $\Delta \kappa_{V}$.

Within the nonlinear realization scenario, there are two operators which contribute to anomalous TGV's (and not to two-point functions) at lowest order [15, [16]. Writing the heavy mass dependence explicitly, they are:

$$
-i g \frac{v^{2}}{\Lambda^{2}} L_{9 L} \operatorname{Tr}\left[W^{\mu \nu} D_{\mu} \Sigma D_{\nu} \Sigma^{\dagger}\right]-i g^{\prime} \frac{v^{2}}{\Lambda^{2}} L_{9 R} \operatorname{Tr}\left[B^{\mu \nu} D_{\mu} \Sigma^{\dagger} D_{\nu} \Sigma\right] .
$$

These are related to $\Delta g_{1}^{V}$ and $\Delta \kappa_{V}$ by:

$$
\begin{aligned}
\Delta g_{1}^{Z} & =\frac{e^{2}}{2 c^{2} s^{2}} \frac{v^{2}}{\Lambda^{2}} L_{9 L}, \\
\Delta \kappa_{\gamma} & =\frac{e^{2}}{2 s^{2}} \frac{v^{2}}{\Lambda^{2}}\left(L_{9 L}+L_{9 R}\right), \\
\Delta \kappa_{Z} & =\frac{e^{2}}{2 c^{2} s^{2}} \frac{v^{2}}{\Lambda^{2}}\left(L_{9 L} c^{2}-L_{9 R} s^{2}\right),
\end{aligned}
$$


where $s^{2} c^{2}=\pi \alpha\left(m_{Z}\right) / \sqrt{2} G_{F} m_{Z}^{2}$. (The $g_{5}^{Z}$ coupling is studied in Ref. [17.) Note that as far as these three TGV's are concerned the linear and the nonlinear realization are obtained from each other by identifying $L_{9 L}=2 f_{W}$ and $L_{9 R}=2 f_{B}$. In particular the correlation between TGV's as given in Eqs. (13) and (14) holds in both frameworks as long as higher-dimensional operators can be neglected.

Anomalous $Z \gamma V$ couplings again originate only from higher order terms in the effective Lagrangian.

\subsection{Form Factors}

Although the anomalous TGV's $\Delta g_{1}^{V}, \Delta \kappa_{V}$, etc. appear as constants in Eqs. (2) and (6), they should rather be considered as form factors. Consider the $\Delta \kappa_{V}$ term, $W_{\mu}^{\dagger} W_{\nu} V^{\mu \nu}$. One can write down similar higher-order terms such as

$$
\frac{1}{\Lambda^{2}} W_{\mu}^{\dagger} W_{\nu} \square V^{\mu \nu}
$$

which has the same Feynman rules as $W_{\mu}^{\dagger} W_{\nu} V^{\mu \nu}$, except for a multiplicative $q^{2}$ dependence due to the derivatives in $\square$. Taking into account all such operators, the overall coefficient of the Feynman rule is not $\Delta \kappa_{V}$, but rather a form factor

$$
\Delta \kappa_{V}^{0}\left(1+a \frac{q^{2}}{\Lambda^{2}}+b\left(\frac{q^{2}}{\Lambda^{2}}\right)^{2}+\ldots\right),
$$

where $a, b$, etc. are $\mathcal{O}(1)$. Since a constant anomalous TGV would lead to unitarity violation at high energies [18] such a form factor behaviour is a feature of any model of anomalous couplings. When studying $W^{+} W^{-}$production at an $e^{+} e^{-}$collider at fixed $q^{2}=s$ this form factor behaviour is of no consequence. Weak boson pair production at hadron colliders, however, probes the TGV's over a large $q^{2}=\hat{s}$ range and is very sensitive to the fall-off of anomalous TGV's which necessarily happens once the threshold of new physics is crossed. Not taking this cutoff into account results in unphysically large cross sections at high energy (which violate unitarity) and thus leads to a substantial overestimate of experimental sensitivities. In our analysis in Section 3 we will assume a simple power law behaviour, e.g.

$$
\Delta \kappa_{V}\left(q^{2}\right)=\frac{\Delta \kappa_{V}^{0}}{\left(1+q^{2} / \Lambda_{F F}^{2}\right)^{n}},
$$

and similarly for the other TGV's. Here $\Lambda_{F F}$ is the form factor scale which is a function of the scale of new physics, $\Lambda$. For $W W V$ couplings we shall use the exponent $n=2$, which will be referred to as the 'dipole form factor' below. For $Z \gamma V$ couplings we choose $n=3(n=4)$ for $h_{3}^{V}\left(h_{4}^{V}\right)$. Due to the form factor behaviour of the anomalous couplings, the experimental limits extracted from hadron collider experiments explicitly depend on $\Lambda_{F F}$.

The values $\Delta \kappa_{V}^{0}$ etc. of the form factors at low energy are constrained by partial wave unitarity of the inelastic vector boson pair production amplitude in fermion 
antifermion annihilation at arbitrary center of mass energies. Assuming that only one anomalous coupling is nonzero at a time, one finds, for $\Lambda_{F F} \gg m_{W}, m_{Z}$ [11, 19]

$$
\begin{array}{ll}
\left|\Delta g_{1}^{Z 0}\right| \leq \frac{n^{n}}{(n-1)^{n-1}} \frac{0.84 \mathrm{TeV}^{2}}{\Lambda_{F F}^{2}}, & \left|g_{5}^{Z 0}\right| \leq \frac{(2 n)^{n}}{(2 n-1)^{n-1 / 2}} \frac{3.2 \mathrm{TeV}}{\Lambda_{F F}}, \\
\left|\Delta \kappa_{\gamma}^{0}\right| \leq \frac{n^{n}}{(n-1)^{n-1}} \frac{1.81 \mathrm{TeV}^{2}}{\Lambda_{F F}^{2}}, & \left|\Delta \kappa_{Z}^{0}\right| \leq \frac{n^{n}}{(n-1)^{n-1}} \frac{0.83 \mathrm{TeV}^{2}}{\Lambda_{F F}^{2}}, \\
\left|\lambda_{\gamma}^{0}\right| \leq \frac{n^{n}}{(n-1)^{n-1}} \frac{0.96 \mathrm{TeV}^{2}}{\Lambda_{F F}^{2}}, & \left|\lambda_{Z}^{0}\right| \leq \frac{n^{n}}{(n-1)^{n-1}} \frac{0.52 \mathrm{TeV}^{2}}{\Lambda_{F F}^{2}}, \\
\left|h_{30}^{Z}\right| \leq \frac{\left(\frac{2}{3} n\right)^{n}}{\left(\frac{2}{3} n-1\right)^{n-3 / 2}} \frac{0.126 \mathrm{TeV}^{3}}{\Lambda_{F F}^{3}}, & \left|h_{30}^{\gamma}\right| \leq \frac{\left(\frac{2}{3} n\right)^{n}}{\left(\frac{2}{3} n-1\right)^{n-3 / 2}} \frac{0.151 \mathrm{TeV}^{3}}{\Lambda_{F F}^{3}}, \\
\left|h_{40}^{Z}\right| \leq \frac{\left(\frac{2}{5} n\right)^{n}}{\left(\frac{2}{5} n-1\right)^{n-5 / 2}} \frac{2.1 \cdot 10^{-3} \mathrm{TeV}^{5}}{\Lambda_{F F}^{5}}, & \left|h_{40}^{\gamma}\right| \leq \frac{\left(\frac{2}{5} n\right)^{n}}{\left(\frac{2}{5} n-1\right)^{n-5 / 2}} \frac{2.5 \cdot 10^{-3} \mathrm{TeV}^{5}}{\Lambda_{F F}^{5}} .
\end{array}
$$

The bounds listed in Eqs. (22) - (26) have been computed with $m_{W}=80 \mathrm{GeV}$ and $m_{Z}=91.1 \mathrm{GeV}$. In order to satisfy unitarity, $n \geq 1$ for $\Delta g_{1}^{Z}, \Delta \kappa_{V}$ and $\lambda_{V}, n \geq 1 / 2$ for $g_{5}^{Z}, n \geq 3 / 2$ for $h_{3}^{V}$, and $n \geq 5 / 2$ for $h_{4}^{V}$. If more than one coupling is varied at a time, cancellations between the TGV's may occur, and the unitarity limits are weakened somewhat. For $\Lambda_{F F} \gg m_{W}, m_{Z}$, the unitarity limits drop like a power of $1 / \Lambda_{F F}$ with increasing values of the form factor scale. The experimental limits obtained from hadron collider experiments must be compared with the bounds derived from $S$ matrix unitarity. Experiments constrain the $W W V$ and $Z \gamma V$ couplings non-trivially only if the experimental limits are more stringent than the unitarity bounds, for a given value of $\Lambda_{F F}$.

Strictly speaking the appearance of form factor effects implies that the effective Lagrangian description in terms of a small set of low-dimensional operators breaks down, i.e. one is probing weak boson pair production at the scale of new physics. New channels are expected to open up as well. However, the corresponding cross sections might be too small to be observable immediately or the experimental signatures might be obscured by backgrounds (compare e.g. WZ production in the Fermi theory). Thus form factors are a tool to extend the use of effective Lagrangians to the entire energy range which is accessible at hadron colliders.

\subsection{Phenomenological Bounds from High Precision Experiments}

In Section 2.2, we have discussed the reasons why anomalous TGV's are expected to be $\mathcal{O}\left(m_{W}^{2} / \Lambda^{2}\right)$ at most in an effective Lagrangian approach. However, it is also interesting to ask what is known about anomalous TGV's from experiment. The errors of present direct measurements, via pair production of electroweak bosons, are still very large (of order 100\%, see Section 3). More precise constraints might then arise from loop contributions to precisely measured quantities such as $(g-2)_{\mu}$ [12, 20], the $b \rightarrow s \gamma$ decay rate [21, 22], $B \rightarrow K^{(*)} \mu^{+} \mu^{-}$[23], the $Z \rightarrow b \bar{b}$ [24] rate and oblique corrections (i.e. corrections to the two point functions) to 4 -fermion $S$-matrix elements. Oblique corrections combine information from the 
recent LEP/SLD data, neutrino scattering experiments, atomic parity violation, $\mu$ decay, and the $W$-mass measurement at hadron colliders. These analyses have been performed for $W W V$ couplings in the context of linear and nonlinear realizations, and we discuss both of these in turn.

\section{- Linear Realization}

A complete analysis of low energy constraints on the coefficients of the effective Lagrangian of Eq. (11) was performed in 1992 [8]. Here we update these results by using the comprehensive 1994 analysis of electroweak data by Hagiwara et al. [25]. With $\alpha=4 \pi \bar{e}^{2}(0)$ taken as an input parameter, the neutral- and chargedcurrent data may be parameterized in terms of three effective form-factors, $\bar{g}_{Z}^{2}\left(q^{2}\right)$ and $\bar{g}_{W}^{2}\left(q^{2}\right)$ defining the coupling strength of the $Z$ and the $W$ at momentum transfer $q$ and the square of the effective weak mixing angle, $\bar{s}^{2}\left(q^{2}\right)$. For $m_{t}=174 \mathrm{GeV}$ and $\alpha_{s}\left(m_{Z}^{2}\right)=0.12$ the LEP and SLD data can be summarized in terms of

$$
\bar{g}_{Z}^{2}\left(m_{Z}^{2}\right)=0.55673 \pm 0.00087, \quad \bar{s}^{2}\left(m_{Z}^{2}\right)=0.23051 \pm 0.00042, \quad \rho=0.28,
$$

where $\rho$ is the correlation of the two values. In a similar fashion the low-energy data on neutrino scattering and atomic parity violation determine the same form-factors at zero momentum transfer:

$$
\bar{g}_{Z}^{2}(0)=0.5462 \pm 0.0036, \quad \bar{s}^{2}(0)=0.2353 \pm 0.0044, \quad \rho=0.53 .
$$

Finally, the $W$-mass measurement at hadron colliders together with the input value of $G_{F}$ can be translated into a measurement of $\bar{g}_{W}^{2}(0)$ :

$$
\bar{g}_{W}^{2}(0)=0.4225 \pm 0.0017 \text {. }
$$

These five measurements are closely related to other formulations of the oblique corrections, like the $S, T$, and $U$ parameters of Peskin and Takeuchi [26]. The

new feature here is the inclusion of the $q^{2}$ dependence of the form - factors [8, 25, 27]. Indeed, new physics contributions like the operators $\mathcal{O}_{D W}$ or $\mathcal{O}_{D B}$ do lead to a nontrivial $q^{2}$ dependence of the form-factors, and the more general analysis is needed to constrain these operators. Low energy bounds are obtained by fitting

$$
\begin{aligned}
S & =S_{S M}\left(m_{t}, m_{H}\right)+\Delta S, \\
T & =T_{S M}\left(m_{t}, m_{H}\right)+\Delta T \text { etc. }
\end{aligned}
$$

to the data. Here the SM contributions $\left(S_{S M}\right.$ etc.) introduce a significant dependence on the values of the Higgs boson and the top quark masses.

The four operators $\mathcal{O}_{D W}, \mathcal{O}_{D B}, \mathcal{O}_{B W}$, and $\mathcal{O}_{\Phi, 1}$ contribute already at tree level,

$$
\begin{aligned}
\Delta \delta \rho & =\alpha \Delta T=-\frac{v^{2}}{2 \Lambda^{2}} f_{\Phi, 1}, \\
\Delta S & =-32 \pi s^{2} \frac{m_{W}^{2}}{\Lambda^{2}}\left(f_{D W}+f_{D B}\right)-4 \pi \frac{v^{2}}{\Lambda^{2}} f_{B W}
\end{aligned}
$$


with similar results for the other form-factors. Fitting these to the five data points one obtains measurements of the coefficients of the operators in the effective Lagrangian. The central values depend on the top quark and Higgs boson masses which we parameterize in terms of

$$
x_{t}=\frac{m_{t}-175 \mathrm{GeV}}{100 \mathrm{GeV}}, \quad x_{H}=\frac{m_{H}}{100 \mathrm{GeV}} .
$$

Within better than $5 \%$ of the $1 \sigma$ errors, and in the ranges $140 \mathrm{GeV}<m_{t}<220 \mathrm{GeV}$ and $60 \mathrm{GeV}<m_{H}<800 \mathrm{GeV}$ this dependence is given by

$$
\begin{aligned}
f_{D W} & =-0.35+0.012 \log x_{H}-0.14 x_{t} \pm 0.62, \\
f_{D B} & =-11+0.11 \log x_{H}-0.58 x_{t} \pm 11, \\
f_{B W} & =3.1+0.072 \log x_{H}+0.22 x_{t} \pm 2.6, \\
f_{\Phi, 1} & =0.23-0.031 \log x_{H}+0.36 x_{t} \pm 0.17,
\end{aligned}
$$

assuming $\Lambda=1 \mathrm{TeV}$. The correlation matrix $C$ is found to be

$$
C=\frac{V_{i j}}{\sqrt{V_{i i} V_{j j}}}=\left(\begin{array}{cccc}
1 . & -0.323 & 0.151 & -0.228 \\
& 1 . & -0.979 & -0.806 \\
& & 1 . & 0.905 \\
& & & 1 .
\end{array}\right) \text {. }
$$

Both the $1 \sigma$ errors and the correlation matrix elements are independent of $m_{H}$ and $m_{t}$ to high precision. Note the strong correlations between the coefficients of the dimension six operators, in particular between $f_{D B}, f_{B W}$ and $f_{\Phi, 1}$. While the contributions of these four operators are already constrained at the tree level, the anomalous $W W V$ couplings only contribute at the 1-loop level to the oblique correction parameters. Neglecting all terms which are not logarithmically divergent for $\Lambda \rightarrow \infty$, the leading effects are given by replacing $f_{D W}$ etc. in Eqs. (32) and (33) by the renormalized quantities

$$
\begin{aligned}
f_{D W}^{r}= & f_{D W}-\frac{1}{192 \pi^{2}} f_{W} \log \frac{\Lambda^{2}}{\mu^{2}} \\
f_{D B}^{r}= & f_{D B}-\frac{1}{192 \pi^{2}} f_{B} \log \frac{\Lambda^{2}}{\mu^{2}} \\
f_{B W}^{r}= & f_{B W}+\frac{\alpha}{32 \pi s^{2}} \log \frac{\Lambda^{2}}{\mu^{2}}\left(f_{B}\left(\frac{20}{3}+\frac{7}{3 c^{2}}+\frac{m_{H}^{2}}{m_{W}^{2}}\right)\right. \\
& \left.\quad-f_{W}\left(4+\frac{1}{c^{2}}-\frac{m_{H}^{2}}{m_{W}^{2}}\right)+12 g^{2} f_{W W W}\right), \\
f_{\Phi, 1}^{r}= & f_{\Phi, 1}+\frac{3 \alpha}{8 \pi c^{2}} \log \frac{\Lambda^{2}}{\mu^{2}}\left(f_{B} \frac{m_{H}^{2}}{v^{2}}+\frac{3 m_{W}^{2}}{v^{2}}\left(f_{B}+f_{W}\right)\right) .
\end{aligned}
$$

Here, $\mu$ denotes the unit-of-mass of the dimensional regularization which has been used to regulate the divergencies which appear in the calculation. The $\log \frac{\Lambda^{2}}{\mu^{2}}$ terms 
Figure 1: Constraints on a) $\Delta \kappa_{\gamma}$ vs. $\lambda$ and b) $\Delta \kappa_{Z}$ vs. $\lambda$ at $95 \%$ confidence level (CL). All coefficients of the dimension six operators in Eq. (11) are assumed to vanish except for a) $f_{B}=f_{W}$ and $f_{W W W}$ and b) $f_{B}=-f_{W}$ and $f_{W W W}$. Correlations are shown for three representative Higgs boson masses.

in Eqs. (40) - (43) describe mixing of the operators between the new physics scale $\Lambda$ and the weak boson mass scale $\mu=m_{W}$.

The results of Eqs. (40) - (43) nicely illustrate the problem of deriving low energy bounds for the TGV's. The dominant contributions of the coefficients $f_{B}$, $f_{W}$ and $f_{W W W}$ are merely renormalizations of those 4 operators which already contribute at tree level. Also, the precision electroweak data are barely sufficient to constrain all four coefficients $f_{D W}^{r}, f_{D B}^{r}, f_{B W}^{r}$, and $f_{\Phi, 1}^{r}$. Hence, indirect bounds on the TGV's are only possible if one makes stringent assumptions on the size of these 'tree level' coefficients. An analogous problem appears when considering 1-loop contributions of the TGV's to $(g-2)_{\mu}, Z \rightarrow b \bar{b}$ or $b \rightarrow s \gamma$ and hence data on those observables do not provide model-independent bounds either.

Nevertheless, one may proceed and assume that cancellations between tree level and 1-loop contributions or between any of the coefficients of the dimension 6 operators are unnatural. In practice one assumes that all $f_{i}$ vanish at the scale of new physics $\Lambda$ except for the one or two whose effect one wants to analyse. The result of such an exercise is shown in Fig. 1. Clearly anomalous TGV's of $\mathcal{O}(1)$ are still allowed by the data. Note that these bounds become more stringent as the Higgs boson mass increases, pointing to more severe bounds in the nonlinear realization scenario. If the top quark mass is varied between $150 \mathrm{GeV}$ and $200 \mathrm{GeV}$, the range allowed for the anomalous couplings increases by up to $50 \%$. 
Other processes which at the 1-loop level are sensitive to anomalous gauge boson couplings also give constraints of $\mathcal{O}(1)$ at best. The current CLEO measurement of the inclusive $b \rightarrow s \gamma$ decay rate [22], for example, still allows $-2.6<\Delta \kappa_{\gamma}<-1.2$ and $-0.5<\Delta \kappa_{\gamma}<0.4$ (for $\lambda_{\gamma}=0$ ), and $-1.7<\lambda_{\gamma}<1.0$ (for $\Delta \kappa_{\gamma}=0$ ) at 95\% CL.

A more stringent assumption on the coefficients of the dimension 6 operators has been proposed by De Rújula et al. [10]. There are no obvious symmetries which distinguish the tree level operators $\mathcal{O}_{B W}, \mathcal{O}_{D W}, \mathcal{O}_{D B}$, and $\mathcal{O}_{\Phi, 1}$ from the remaining ones. For a generic model of the underlying dynamics one may hence expect e.g. $\left|f_{B}+f_{W}\right| \approx\left|f_{B W}\right|$ which with $f_{B W} / \Lambda^{2}=(3.1 \pm 2.6) \mathrm{TeV}^{-2}$ implies $\left|\Delta \kappa_{\gamma}\right|=$ $\left|f_{B}+f_{W}\right| m_{W}^{2} / 2 \Lambda^{2}<0.03$ at " $95 \%$ CL", a value too small to be observable in $W^{+} W^{-}$ production at LEP II, but still in the interesting range for future linear colliders. Although this naturalness argument is compelling, it is clearly not a proof that anomalous TGV's are indeed small.

- Nonlinear Realization

There are several analyses in the literature which discuss the bounds on anomalous TGV's in the context of the nonlinear realization scenario [16, 28, 29]. All conclusions are quite similar. The limits obtained in the nonlinear realization framework are very similar to those obtained in the linear realization scenario for a large Higgs boson mass. In the following, we will briefly review the results of Ref. 28].

As in the linear realization case the effective Lagrangian is nonrenormalizable, and therefore the loop diagrams diverge. Conceptually, this is not a problem - the effective Lagrangian already contains an infinite number of terms, so one can just add a counterterm to cancel the infinities found in any loop calculation. In other words, if an anomalous TGV contributes at loop level to an observable, the divergence of the calculation just renormalizes the coefficient of that observable. At the calculational level, however, one has to decide how to deal with the infinities. In the past it was common to simply use a cutoff $\tilde{\Lambda}$ to regulate the divergence. With this technique one often obtained a cutoff dependence of the form $\tilde{\Lambda}^{2}$ or even $\tilde{\Lambda}^{4}$, resulting in extremely stringent constraints on anomalous TGV's. However, it was argued in Ref. [6] that the use of cutoffs was incorrect, and often gave misleading results. Instead the authors of Ref. [6] advocate the use of dimensional regularization, along with the decoupling-subtraction renormalization scheme. This is the procedure used in Ref. [28].

For the calculational details, we refer the reader to Ref. [28]. Here we only present the results of the global fit. First, consider the case in which only one of the anomalous TGV couplings, $\Delta g_{1}^{Z}, \Delta \kappa_{V}$ and $\lambda_{V}$, is nonzero. (The coupling $g_{5}^{Z}$ was not considered in this paper.) The fit gave the following constraints at $1 \sigma$ :

$$
\begin{aligned}
\Delta g_{1}^{Z} & =-0.033 \pm 0.031 \\
\Delta \kappa_{\gamma} & =0.056 \pm 0.056, \\
\Delta \kappa_{Z} & =-0.0019 \pm 0.044, \\
\lambda_{\gamma} & =-0.036 \pm 0.034,
\end{aligned}
$$




$$
\lambda_{Z}=0.049 \pm 0.045
$$

If taken at face value, these limits would imply that most anomalous TGV's are too small to be seen at LEP II or in future Tevatron experiments (see Section 3). The LHC and NLC, on the other hand, will be able to improve these bounds considerably.

However, one should keep in mind that these bounds are rather artificial. It is very hard to imagine that physics beyond the SM will produce only one anomalous TGV. In general, all such couplings will be produced. In a fit to all five anomalous couplings simultaneously, the constraints virtually disappear, due to the possibility of cancellations. At best, one can only conclude that the anomalous TGV couplings are less than $\mathcal{O}(1)$ and even here one must assume that tree level contributions do not cancel the TGV effects.

Even so, the bounds of Eq. (44) are interesting. These values represent the sensitivity of the global fit of electroweak data to specific anomalous couplings. Once all of the couplings are allowed to vary simultaneously, no significant bound remains. This obviously implies that, in that part of the allowed region for which the TGV couplings are large, cancellations occur among the contribution of the various anomalous couplings to low-energy observables. Equation (44) gives an indication of the level of cancellation required to account for the low-energy data in the event that an anomalous TGV at the $10 \%$ level is discovered.

\subsection{Summary}

We have discussed our theoretical understanding of, and the phenomenological constraints from high precision experiments on, the anomalous TGV's of Eqs. (1) and (2). The phenomenological effective Lagrangian describing anomalous couplings appears not to respect the $S U(2)_{L} \times U(1)_{Y}$ gauge symmetry. However, it can be interpreted as the unitary-gauge expression of an effective Lagrangian in which the $S U(2)_{L} \times U(1)_{Y}$ symmetry is manifest. How this symmetry is realized depends on the particle content of the effective Lagrangian. Regardless of how one realizes the $S U(2)_{L} \times U(1)_{Y}$ gauge symmetry, the anomalous TGV's $\Delta g_{1}^{Z}, \Delta \kappa_{V}, \lambda_{V}$ and $g_{5}^{Z}$ are expected to be at most $\mathcal{O}\left(m_{W}^{2} / \Lambda^{2}\right)$, where $\Lambda$ is the scale of new physics. (In the nonlinear-realization scenario, $\lambda_{V}$ is $\mathcal{O}\left(m_{W}^{4} / \Lambda^{4}\right)$ ). $Z \gamma V$ couplings are at most $\mathcal{O}\left(m_{Z}^{4} / \Lambda^{4}\right)$. Thus, for $\Lambda \sim 1 \mathrm{TeV}$ anomalous TGV's of $\mathcal{O}\left(10^{-2}\right)$ or less are expected. The discovery of larger anomalous TGV's at present or future colliders would indicate that the new physics responsible for them originates below the $1 \mathrm{TeV}$ scale. It is therefore likely, though not certain, that the new physics will first be found directly, rather than through (indirect) contributions to anomalous TGV's.

There is indirect evidence from constraints on oblique correction parameters (2-point functions) that anomalous TGV's are indeed $\lesssim \mathcal{O}\left(10^{-2}\right)$. The limits obtained from these constraints, however, do depend strongly on other parameters, such as the Higgs boson and top quark mass (in the framework where the electroweak symmetry is realized linearly; see Fig. 1). They also strongly depend on naturalness arguments which, though compelling, cannot be considered a proof that large anomalous TGV's do not exist. Strictly speaking, anomalous TGV's are 
unconstrained by the electroweak precision data since the possibility of large cancellations cannot be excluded. Thus, it is necessary for experiments to search directly for evidence of anomalous TGV's, even though, in light of our current theoretical understanding, such experiments will likely yield null results.

\section{Measuring $W W V$ and $Z \gamma V$ Couplings in Collider Experiments}

\subsection{General Overview}

In this Section we discuss possibilities to measure the $W W V$ and $Z \gamma V$ couplings directly in collider experiments. To simplify our discussion, we assume that $g_{1}^{\gamma}=1$ and $g_{5}^{\gamma}=g_{5}^{Z}=0$ in the following. As we have mentioned in the Introduction, electromagnetic gauge invariance requires $g_{1}^{\gamma}=1$ and $g_{5}^{\gamma}=0$ for on-shell photons. In contrast to the other couplings, $g_{5}^{V}, V=\gamma, Z$, violates charge conjugation and parity. Possibilities to measure $g_{5}^{Z}$ in $e^{+} e^{-}$collisions are discussed in Ref. 17].

At hadron colliders (Tevatron, LHC), di-boson production offers the best opportunity to probe the $W W V$ and $Z \gamma V$ vertices. The generic set of Feynman diagrams contributing to di-boson production is shown in Fig. 2. Whereas $W^{+} W^{-}$ production is sensitive to $W W \gamma$ and $W W Z$ couplings, only the $W W \gamma(W W Z)$ vertex is tested in $W^{ \pm} \gamma\left(W^{ \pm} Z\right)$ production. $Z \gamma V$ couplings are probed in $p p, p \bar{p} \rightarrow Z \gamma$. In order to reduce the QCD background, one has to require that at least one of the $W$ and/or $Z$ bosons decays leptonically. In $p p, p \bar{p} \rightarrow W^{+} W^{-}, t \bar{t}$ production represents an additional background. $W^{ \pm} \gamma$ and $W^{ \pm} Z$ production are of special interest due to the presence of amplitude zeros [30, 31].

Electroweak boson pair production at hadron colliders will be discussed in detail in Section 3.2. We present the general strategy in extracting information on three vector boson couplings, summarize the current limits on $W W V$ and $Z \gamma V$ couplings from $\mathrm{CDF}$ and $\mathrm{D} \varnothing$, and investigate the prospects of measuring these couplings in future Tevatron and LHC experiments. We also discuss possibilities to search for the amplitude zeros in $W^{ \pm} \gamma$ and $W^{ \pm} Z$ production.
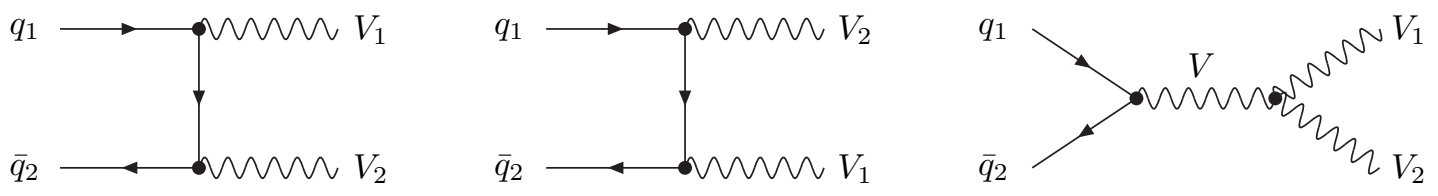

Figure 2: Generic Feynman diagrams contributing to di-boson production in hadronic collisions. $V, V_{1}, V_{2}=W, \gamma, Z$.

At LEP, $Z \gamma V$ couplings can be tested in single photon production $\left(e^{+} e^{-} \rightarrow\right.$ $\bar{\nu} \nu \gamma)$ and radiative $Z$ decays. Single photon production, in principle, is also sensitive to the $W W \gamma$ vertex [32]. $W W Z$ couplings can be probed in the rare decay $Z \rightarrow$ $W f \bar{f}^{\prime}$ [33]. In both cases, however, the sensitivity is not sufficient to compete with the existing limits from CDF and $\mathrm{D} \varnothing$ (see below). The constraints on $Z \gamma V$ 
couplings from LEP experiments will be discussed in Section 3.3.1. At LEP II, $e^{+} e^{-} \rightarrow W^{+} W^{-}$and $e^{+} e^{-} \rightarrow Z \gamma$ are the prime reactions to test $W W V$ and $Z \gamma V$ couplings (see Section 3.3.2). $W$ pair production at a linear $e^{+} e^{-}$collider with a center of mass energy of $500 \mathrm{GeV}$ or more ["Next Linear Collider" (NLC)] will be discussed in Section 3.3.3. Using laser backscattering [34, the NLC can also be operated as a $e \gamma$ or $\gamma \gamma$ collider, with a center of mass energy of up to $\sim 80 \%$ of that available in the $e^{+} e^{-}$mode, and comparable luminosity. This opens the possibility of testing the $W W V$ couplings in processes such as $e \gamma \rightarrow W \nu$ [35, 36], or $\gamma \gamma \rightarrow W^{+} W^{-}$[36, 37] in addition to $e^{+} e^{-} \rightarrow W^{+} W^{-} . \quad Z \gamma V$ couplings can be investigated in $Z \gamma$ production and in $\gamma e \rightarrow Z e$ [38]. The NLC could even be operated as an $e^{-} e^{-}$collider. Possibilities to probe the three vector boson couplings in $e^{-} e^{-}$ collisions have been explored in Ref. [39]. The limits on anomalous $W W V$ couplings expected from reactions accessible in $e \gamma, \gamma \gamma$ and $e^{-} e^{-}$collisions are similar to those from $e^{+} e^{-} \rightarrow W^{+} W^{-}$. Alternative $e^{+} e^{-}$processes, such as $e^{+} e^{-} \rightarrow e^{+} e^{-} W^{+} W^{-}$, $W^{+} W^{-} V(V=\gamma, Z)$, or $\bar{\nu} \nu Z$ are significantly less sensitive to three gauge boson couplings than $W$ pair production. For a summary of these modes see Ref. 40. The sensitivity bounds obtained from $e^{+} e^{-} \rightarrow W^{+} W^{-}$are therefore representative for the limits on anomalous gauge boson couplings which can be achieved at the NLC.

The $W W V$ couplings can, in principle, also be tested in single $W$ and $Z$ production at HERA [41]. However, in order to achieve limits which are comparable to the current $\mathrm{CDF} / \mathrm{D} \varnothing$ bounds (see Section 3.2.2), integrated luminosities of the order $1 \mathrm{fb}^{-1}$ are needed. Since it is not expected that those can be achieved within the next few years, anomalous gauge boson couplings at HERA will not be discussed in this report.

\subsection{Di-boson Production at Hadron Colliders}

\subsubsection{Theoretical Background}

From the phenomenological effective Lagrangian [see Eqs. (11) and (6)] it is straightforward to derive cross section formulas for the di-boson production processes,

$$
q \bar{q} \rightarrow W^{+} W^{-}, Z \gamma
$$

and

$$
q \bar{q}^{\prime} \rightarrow W^{ \pm} \gamma, W^{ \pm} Z
$$

For our subsequent discussion we find it convenient to briefly discuss the contributions of anomalous couplings to the helicity amplitudes of the processes listed in (45) and (46). In $q \bar{q}^{\prime} \rightarrow W \gamma$, for example, the anomalous contributions $\Delta \mathcal{M}_{\lambda^{\gamma} \lambda^{W}}$, ( $\lambda^{\gamma}$ and $\lambda^{W}$ are the photon and $W$ helicities, respectively) to the helicity amplitudes are given by 42

$$
\Delta \mathcal{M}_{ \pm 0}=\frac{e^{2}}{\sin \theta_{W}} \frac{\sqrt{\hat{s}}}{2 m_{W}}\left(\Delta \kappa_{\gamma}+\lambda_{\gamma}\right) \frac{1}{2}(1 \mp \cos \Theta)
$$




$$
\Delta \mathcal{M}_{ \pm \pm}=\frac{e^{2}}{\sin \theta_{W}} \frac{1}{2}\left(\frac{\hat{s}}{m_{W}^{2}} \lambda_{\gamma}+\Delta \kappa_{\gamma}\right) \frac{1}{\sqrt{2}} \sin \Theta
$$

where $\Theta$ denotes the scattering angle of the photon with respect to the quark direction, measured in the $W \gamma$ rest frame, and $\sqrt{\hat{s}}$ is the invariant mass of the $W$-photon system. Similar expressions can be derived for the anomalous contributions to the $W Z, W^{+} W^{-}$and $Z \gamma$ helicity amplitudes.

While the SM contribution to the di-boson amplitudes is bounded from above for fixed scattering angle $\Theta$, the anomalous contributions rise without limit as $\hat{s}$ increases, eventually violating unitarity. This is the reason the anomalous couplings must show a form factor behavior at very high energies (see Section 2.3). Anomalous values of $\lambda_{V}, V=\gamma, Z$, are enhanced by $\hat{s} / m_{W}^{2}$ in the amplitudes $\mathcal{M}_{ \pm \pm}$for all di-boson production processes. Terms containing $\Delta \kappa_{V}$ mainly contribute to $\mathcal{M}_{ \pm 0}$ in $W V$ production and grow only with $\sqrt{\hat{s}} / m_{W}$. In $q \bar{q} \rightarrow W^{+} W^{-}$, on the other hand, the $\Delta \kappa_{V}$ term mostly contributes to the $(0,0)$ amplitude and is enhanced by a factor $\hat{s} / m_{W}^{2}[3]$. Non-standard values of $\Delta g_{1}^{Z}$ mostly affect the $(0,0)[( \pm, 0)$ and $(0, \pm)]$ amplitude in $W Z\left[W^{+} W^{-}\right]$production, and are proportional to $\hat{s} / m_{W}^{2}$ $\left[\sqrt{\hat{s}} / m_{W}\right]$ [3, 43]. The best limits on $\Delta \kappa_{V}\left(\Delta g_{1}^{Z}\right)$ are therefore expected from $q \bar{q} \rightarrow$ $W^{+} W^{-}\left(q \bar{q}^{\prime} \rightarrow W Z\right)$. In $Z \gamma$ production, terms proportional to $h_{3}^{V}\left(h_{4}^{V}\right)$ grow like $\left(\sqrt{\hat{s}} / m_{Z}\right)^{3}\left(\left(\sqrt{\hat{s}} / m_{Z}\right)^{5}\right)[19]$.

For large values of the di-boson invariant mass $\sqrt{\hat{s}}$, the non-standard contributions to the helicity amplitudes would dominate, and would suffice to explain differential distributions of the photon and the $W / Z$ decay products. Due to the fact that anomalous couplings only contribute via $s$-channel $W, Z$ or photon exchange, their effects are concentrated in the region of small vector boson rapidities, and the transverse momentum distribution of the vector boson should be particularly sensitive to non-standard $W W V$ and $Z \gamma V$ couplings. This is demonstrated in Fig. 3, where we show the photon $p_{T}$ distribution in $p \bar{p} \rightarrow W^{+} \gamma \rightarrow e^{+} \nu_{e} \gamma$, and the $Z$ boson transverse momentum distribution in $p \bar{p} \rightarrow W^{+} Z \rightarrow \ell_{1}^{+} \nu_{1} \ell_{2}^{+} \ell_{2}^{-}, \ell_{1,2}=e, \mu$, at the Tevatron for the SM and various anomalous $W W V$ couplings. A dipole form factor (see Section 2.3) with scale $\Lambda_{F F}=1 \mathrm{TeV}$ has been assumed. Only one coupling is assumed to deviate from the SM at a time. To simulate detector response, the following cuts have been imposed in Fig. 3:

$$
\begin{array}{rlrl}
p_{T}(\gamma) & >10 \mathrm{GeV}, & & |\eta(\gamma)|<1, \\
p_{T}(\ell)>20 \mathrm{GeV}, & & |\eta(\ell)|<2.5, \quad \ell=e, \mu, \\
p_{T} & >20 \mathrm{GeV}, & & \Delta R(\ell, \ell)>0.4, \\
m_{T}\left(\ell \gamma ; p_{T}\right) & >90 \mathrm{GeV}, & & \Delta R(\gamma, \ell)>0.7 .
\end{array}
$$

Here, $\not_{T}$ denotes the missing transverse momentum, $\eta$ the pseudorapidity, $\Delta R=$ $\left[(\Delta \phi)^{2}+(\Delta \eta)^{2}\right]^{1 / 2}$ the separation in the pseudorapidity - azimuthal angle plane, and $m_{T}$ is the cluster transverse mass defined by

$$
m_{T}^{2}\left(\ell \gamma ; p_{T}\right)=\left[\left(m(\ell \gamma)^{2}+\left|\mathbf{p}_{T}(\ell \gamma)\right|^{2}\right)^{1 / 2}+p_{T}\right]^{2}-\left|\mathbf{p}_{T}(\ell \gamma)+\not \boldsymbol{p}_{T}\right|^{2}
$$


Figure 3: The differential cross section for the transverse momentum of a) the photon in $p \bar{p} \rightarrow W^{+} \gamma$, and b) of the $Z$ boson in $p \bar{p} \rightarrow W^{+} Z$ at the Tevatron in the SM case (solid line) and for various anomalous $W W V$ couplings. The cuts imposed are described in the text.

with $m(\ell \gamma)$ being the $\ell \gamma$ invariant mass. The large lepton photon separation and the $m_{T}$ cut together strongly suppress photon radiation from the final state lepton line (radiative $W$ decays) [42].

Information on anomalous $W W V$ and $Z \gamma V$ couplings can be obtained by comparing the shape of the measured and predicted $p_{T}$ distribution, provided that the signal is not overwhelmed by background. If the background is much larger than the SM prediction, limits on anomalous couplings can still be extracted if a phase space region can be selected where the effects of non-standard three vector boson couplings dominate.

Besides di-boson production, radiative $W(Z)$ decays are also sensitive to $W W \gamma(Z \gamma V)$ couplings. However, the parton center of mass energy in these processes is restricted to values around $\sqrt{\hat{s}}=m_{W}\left(m_{Z}\right)$, and the expected limits on anomalous couplings are significantly worse than those obtained from $W \gamma$ and $Z \gamma$ production where much larger values of $\sqrt{\hat{s}}$ are accessible.

3.2.2 Di-boson Production at the Tevatron: Current Results and Future Prospects

Both, the CDF and D Collaboration have searched for $W \gamma$ 44, 45], $Z \gamma$ 46, 47], $W^{+} W^{-}$[48, 49], and $W Z$ [48 production in the data samples accumulated in run 1a. CDF has also searched for $W \gamma$ and $Z \gamma$ events in the data of the $1988-89$ run [50]. For a recent summary of electroweak boson pair production results from $\mathrm{CDF}$ and $\mathrm{D} \varnothing$ see Ref. [51]. 
CDF (DØ) extract $W \gamma / Z \gamma$ data samples from inclusive $e / \mu$ channel $W / Z$ samples by requiring an isolated photon in a fiducial region of their central (central + endcap) electromagnetic (EM) calorimeters with $E_{T}(\gamma) \geq 7$ (10) GeV. A minimum lepton - photon angular separation of $\Delta R(\ell \gamma)>0.7$ suppresses final-state QED bremsstrahlung. To reduce the QCD background from $W / Z+$ jets production, excess calorimeter transverse energy, $E_{T}$, within a cone of $\Delta R=0.4$ centered on the photon was required to be less than $15 \%(10 \%)$ of the photon $E_{T}$. CDF also required the sum of the transverse momenta of all charged tracks within this cone to be less than $2 \mathrm{GeV} / \mathrm{c}$, and also rejected events with a track pointing directly at the EM cluster. Both experiments required transverse/longitudinal EM shower development consistent with a single photon. The selection criteria yield 25 (23) $W \gamma$ and 8 (6) $Z \gamma$ candidate events for CDF (DØ).

The level of $W / Z+$ jet background, where a jet "fakes" an isolated photon, in each of the $W \gamma / Z \gamma$ data samples is determined by use of QCD jet data samples to obtain a jet misidentification probability $\mathcal{P}_{j \rightarrow \gamma}\left(E_{T}\right)$. For the photon selection criteria used by $\mathrm{CDF}, \mathcal{P}_{j \rightarrow \gamma}\left(E_{T}=9 \mathrm{GeV}\right) \sim 8 \times 10^{-4}$, decreasing exponentially to $\mathcal{P}_{j \rightarrow \gamma}\left(E_{T}=25 \mathrm{GeV}\right) \sim 10^{-4}$, whereas for the photon selection criteria used by D $\varnothing$, $\mathcal{P}_{j \rightarrow \gamma}\left(E_{T}\right) \sim 4 \times 10^{-4}\left(6 \times 10^{-4}\right)$ in the central (endcap) calorimeter, and varies only slowly with $E_{T}$. The jet fragmentation probability distribution was then convoluted with the jet $E_{T}$ spectrum in each of the inclusive $W / Z$ data samples. The $Z \gamma$ background in the $W \gamma$ data arising from non-observation of one of the $Z$ decay leptons is estimated from Monte Carlo simulations. The contributions to $W \gamma$ and $Z \gamma$ production from $W / Z$ decays into $\tau$ leptons are also estimated from MC simulations and found to be small.

The SM cross sections for $W^{+} W^{-}, W^{ \pm} Z$ and $Z Z$ productionf at the Tevatron, including NLO QCD corrections [52], are $9.5 \mathrm{pb}, 2.5 \mathrm{pb}$ and $1.4 \mathrm{pb}$, respectively. Decay modes where one of the weak bosons decays hadronically have significantly larger branching ratios than all leptonic decays:

$$
\begin{aligned}
\operatorname{Br}\left(W W \rightarrow e \nu_{e} e \nu_{e}, \mu \nu_{\mu} \mu \nu_{\mu}\right)=2.4 \%, & B r\left(W W \rightarrow e \nu_{e} \mu \nu_{\mu}\right)=2.4 \%, \\
\operatorname{Br}\left(W Z \rightarrow \ell_{1} \nu_{1} \ell_{2}^{+} \ell_{2}^{-}\right)=1.5 \%, & \ell_{1,2}=e, \mu, \\
\operatorname{Br}(W W \rightarrow \ell \nu j j)=29 \%, & \ell=e, \mu, \\
\operatorname{Br}(W Z \rightarrow \ell \nu j j)=15 \%, & \operatorname{Br}\left(W Z \rightarrow j j \ell^{+} \ell^{-}\right)=4.5 \%, \\
B R\left(Z Z \rightarrow \ell^{+} \ell^{-} j j\right)=9.4 \%, & \operatorname{Br}\left(Z Z \rightarrow \ell_{1}^{+} \ell_{1}^{-} \ell_{2}^{+} \ell_{2}^{-}\right)=0.4 \% .
\end{aligned}
$$

Due to the larger cross section and branching ratio, the $\ell \nu j j$ final state is completely dominated by $W^{+} W^{-}$production. $W^{ \pm} Z$ and $Z Z$ production contribute approximately equally to the $\ell^{+} \ell^{-} j j$ final state. All semihadronic channels suffer from a large $W / Z+$ jets background. $t \bar{t}$ production contributes non-negligibly to the background for $W^{+} W^{-}$production. In contrast, the $\ell_{1} \nu_{1} \ell_{2}^{+} \ell_{2}^{-}$final state is relatively background free.

${ }^{\ddagger} Z Z$ production is, in principle, sensitive to $Z Z V, V=\gamma, Z$ couplings, which vanish in the SM at tree level [3]. We will not discuss the $Z Z V$ couplings accessible in $Z Z$ production in this report. 
$W^{+} W^{-}$and $W Z$ data samples are also extracted from inclusive $e / \mu W / Z$ data. $\mathrm{CDF}$ has analyzed the $W W, W Z \rightarrow \ell \nu j j$ and $Z W \rightarrow \ell^{+} \ell^{-} j j(\ell=e$ or $\mu)$ channels using standard $W / Z$ lepton selection cuts, and requiring $60 \mathrm{GeV} / \mathrm{c}^{2}<m(j j)<110 \mathrm{GeV} / \mathrm{c}^{2}$. For leptonic $W(Z)$ events, CDF eliminates $W / Z+$ jets background events by requiring $p_{T}(j j)>130(100) \mathrm{GeV} / \mathrm{c}$, which also eliminates the SM signal but retains good sensitivity for non-zero $W W V$ anomalous couplings. One event passes the cuts in the $\ell \nu j j$ channel. In the $\ell^{+} \ell^{-} j j$ channel no events survive. A clean candidate event for $p \bar{p} \rightarrow W^{+} Z \rightarrow e^{+} \nu_{e} e^{+} e^{-}$has also been observed in the CDF data set 48]. D $\varnothing$ has analyzed the $W W \rightarrow \ell_{1} \nu_{1} \ell_{2} \nu_{2}, \ell_{1,2}=e, \mu$, channels using standard lepton cuts for selection of $W$ pairs. The $Z$ mass region in the ee channel, $77 \mathrm{GeV} / \mathrm{c}^{2}<m(e e)<105 \mathrm{GeV} / \mathrm{c}^{2}$, is excluded. To suppress the $Z \rightarrow \mu^{+} \mu^{-}$background, a cut of $\not_{T}^{\eta}>30 \mathrm{GeV}$ is imposed, where $\not_{T}^{\eta}$ is the projection of the missing $E_{T}$ vector onto the bisector of the decay angles of the two muons. To reduce the $t \bar{t}$ background, the total hadronic transverse energy in the event is required to be less than $40 \mathrm{GeV}$. Backgrounds from $Z$ decay and fake electrons are estimated from

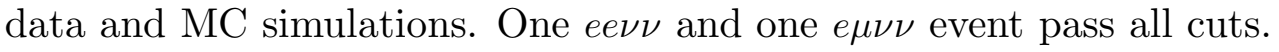

SM and anomalous coupling predictions for the $W \gamma$ and $Z \gamma$ processes are obtained using the event generators of Ref. [42] and [19], and detailed detector simulations. MRSD-' structure functions [53] are used for event generation as they best match the recent $W$ lepton asymmetry measurements from CDF [54]. SM and anomalous coupling predictions for $W^{+} W^{-}$and $W Z$ production are obtained using the event generator of Ref. [55] and MC detector simulations. Presently, a complete calculation of the di-boson transverse momentum distribution, including soft gluon resummation effects, does not exist, except for the $Z Z$ case [56]. Higher order QCD corrections are therefore approximated in the experimental analysis by a $k$-factor and by smearing the transverse momentum of the di-boson system according to the experimentally determined $W / Z$ boson $p_{T}$ spectrum.

Direct experimental limits on $W W \gamma$ and $Z \gamma V$ anomalous couplings for the $W \gamma / Z \gamma$ processes are obtained via binned maximum likelihood fits to the $E_{T}(\gamma)$ distribution. The observed $E_{T}(\gamma)$ distribution is compared to the sum of expected signal plus background(s) prediction, calculating the Poisson likelihood that this sum would fluctuate to the observed number of events in each $E_{T}$ bin, and convoluting with a Gaussian distribution to take into account systematic uncertainties associated with backgrounds, luminosity normalization, structure function choice, $Q^{2}$-scale and uncertainties in the shape of the $p_{T}(W \gamma / Z \gamma)$ distribution, efficiencies, etc. The 95\% CL CDF [44] and DØ 45] limits on anomalous $W W \gamma$ couplings from $W \gamma$ production are shown in Fig. 4a. The bounds on $\Delta \kappa_{\gamma}^{0}$ and $\lambda_{\gamma}^{0}$ extracted by DØ (solid curve) are about $20 \%$ better than those obtained by CDF (short dashed curve). For comparison, we have also included the limits obtained by UA2 [57, and CDF from the 1988-89 data [50]. Due to the smaller center of mass energy $(\sqrt{s}=630 \mathrm{GeV})$, the correlations between the two couplings at the CERN $p \bar{p}$ collider are much more pronounced than at Tevatron energies. The bounds obtained from the 1992-93 data have been obtained using a dipole form factor with scale $\Lambda_{F F}=1.5 \mathrm{TeV}$. The CDF limits from the 1988-89 data are for $\Lambda_{F F}=1 \mathrm{TeV}$. 
Figure 4: Present limits on anomalous $W W V$ couplings from hadron collider experiments.

CDF extracts direct experimental limits on $W W \gamma$ and $W W Z$ anomalous couplings from the $\ell \nu j j$ and $\ell^{+} \ell^{-} j j$ final states via comparison of observed events to the expected signal within cuts, including systematic uncertainties due to luminosity normalization, jet energy scale and resolution, structure function choice and higher order QCD corrections, etc. DØ extracts direct experimental limits on $W W V$ anomalous couplings from the $W W \rightarrow \ell_{1} \nu_{1} \ell_{2} \nu_{2}$ mode via comparison of their $95 \%$ CL upper limit of $\sigma(W W)_{\text {expt }}<91 \mathrm{pb}$ with $\sigma(W W)_{\text {pred }}$ as a function of anomalous couplings.

The limits obtained from $W^{+} W^{-} \rightarrow \ell_{1} \nu_{1} \ell_{2} \nu_{2}$ and $W W, W Z \rightarrow \ell \nu j j$ are summarized and compared to those obtained from $W \gamma$ production in Fig. 4b. In extracting limits on non-standard $W W V$ couplings from $W$ pair production, CDF (DØ) assumed a dipole form factor with scale $\Lambda_{F F}=1.5 \mathrm{TeV}(0.9 \mathrm{TeV}), \Delta \kappa_{\gamma}^{0}=\Delta \kappa_{Z}^{0}, \lambda_{\gamma}^{0}=\lambda_{Z}^{0}$, and $\Delta g_{1}^{Z}=0$. Due to the selection of a phase space region which is particularly sensitive to $W W V$ couplings and the larger branching ratios for $W W, W Z \rightarrow \ell \nu j j$, the bounds obtained from the semihadronic $W W$ and $W Z$ final states are significantly stronger than those found from analyzing the $W W \rightarrow \ell_{1} \nu_{1} \ell_{2} \nu_{2}$ channel. In Section 3.2.1 we have mentioned that the contributions to the $W^{+} W^{-}$helicity amplitudes proportional to $\Delta \kappa_{V}$ grow like $\hat{s} / m_{W}^{2}$ whereas the $\Delta \kappa_{\gamma}$ terms in the $W \gamma$ amplitudes are proportional to $\sqrt{\hat{s}} / m_{W}$. In contrast, the $\lambda_{V}$ terms always grow like $\hat{s} / m_{W}^{2}$. This explains why the limit on $\Delta \kappa_{V}^{0}$ obtained from the semihadronic $W W$ and $W Z$ final states is significantly better than that found from $p \bar{p} \rightarrow W \gamma$ while the bounds on $\lambda_{V}^{0}$ from $W W, W Z \rightarrow \ell \nu j j$ and $W \gamma$ production are almost identical.

Limits on $\Delta \kappa_{\gamma}^{0}$ and $\lambda_{\gamma}^{0}$ extracted from $W \gamma$ production have the advantage of being independent of assumptions about the $W W Z$ vertex. Similarly, information on the $W W Z$ couplings, independent from assumptions on the $W W \gamma$ couplings, can be obtained from $W Z$ production. From the $W Z \rightarrow j j \ell^{+} \ell^{-}$channel, CDF finds 48 
Figure 5: Comparison of current experimental bounds on $W W V$ couplings and limits obtained from $S$-matrix unitarity for a dipole form factor.

$-8.6<\Delta \kappa_{Z}^{0}<9.0$ for $\Delta g_{1}^{Z 0}=\lambda_{Z}^{0}=0$ and $-1.7<\lambda_{Z}^{0}<1.7$ for $\Delta g_{1}^{Z 0}=\Delta \kappa_{Z}^{0}=0$. These limits were obtained for a form factor scale $\Lambda_{F F}=1.5 \mathrm{TeV}$. The $Z Z \rightarrow j j \ell^{+} \ell^{-}$cross section was assumed to be given by the SM prediction.

In Section 2.3 we have seen that constraints from $S$-matrix unitarity severely restrict the values of the low energy anomalous couplings allowed. For sufficiently small values of the form factor scale, the experimental limits on non-standard three vector boson couplings are substantially better than those found from $S$-matrix unitarity [see Eqs. (22) - (26)]. However, for $\Lambda_{F F} \gg m_{W}$, the unitarity bounds decrease like $1 / \Lambda_{F F}^{n}$, with $n=1,2$ for the $W W V$ couplings, and $n=3,5$ for the $Z \gamma V$ couplings whereas the experimental limits depend less sensitively on $\Lambda_{F F}$ [50]. This implies that for sufficiently large form factor scales unitarity bounds eventually will be stronger than the limits extracted from experimental data. In Fig. 5a we compare the current experimental limits on $W W \gamma$ couplings from $W \gamma$ production with the bounds derived from unitarity for $\Lambda_{F F}=1.5 \mathrm{TeV}$. In Fig. 5b a similar comparison is carried out for $W W / W Z \rightarrow \ell \nu j j$ with $\Lambda_{F F}=1.5 \mathrm{TeV}$, and $W W \rightarrow \ell_{1} \nu_{1} \ell_{2} \nu_{2}$ with $\Lambda_{F F}=0.9 \mathrm{TeV}$. These values of $\Lambda_{F F}$ were chosen just large enough that the unitarity bounds would approach the experimental limits. One concludes that the maximum scale which can be probed with the current experimental data on $W \gamma, W W$ and $W Z$ production is of order $1.5-2 \mathrm{TeV}$.

The current CDF [46] and DØ [47] 95\% CL limit contours for anomalous $Z Z \gamma$ couplings are shown in Fig. 6, together with the constraints from $S$-matrix unitarity. The limit contours for $Z \gamma \gamma$ couplings are similar. For completeness, we have also included the CDF result from the 1988-89 run [50]. The D $\varnothing$ limits on $h_{30}^{V}$ and $h_{40}^{V}$ are about $30 \%$ more stringent than those obtained by CDF. In order to derive these limits, generalized dipole form factors with $\Lambda_{F F}=0.5 \mathrm{TeV}$, and powers 
Figure 6: Present limits on anomalous $Z Z \gamma$ couplings from hadron collider experiments, and constraints from $S$-matrix unitarity.

$n=3(n=4)$ for $h_{3}^{V}\left(h_{4}^{V}\right)$, are assumed (see Section 2.3). Since the anomalous contributions to the $Z \gamma$ helicity amplitudes grow faster with energy than those in $W \gamma$ production, the experimental limits on $h_{30}^{V}$ and $h_{40}^{V}$ depend rather sensitively on the form factor scale chosen. The maximum form factor scale which can be probed in $Z \gamma$ production with present experimental data is $\Lambda_{F F} \approx 500 \mathrm{GeV}$.

Table 1 summarizes the current results on anomalous $W W V$ and $Z \gamma V$ couplings from hadron colliders. With the limited statistics of di-boson events currently available, deviations from the SM cross section have to be large at least in some regions of phase space in order to lead to an observable effect. The best direct limits on $\Delta \kappa_{V}^{0}$ are currently obtained from the $\ell \nu j j$ final state. $W \gamma$ production results in somewhat better bounds on $\lambda_{\gamma}^{0}$ than $p \bar{p} \rightarrow W W, W Z \rightarrow \ell \nu j j$. So far, no attempt has been made to combine the limits of CDF and $D \varnothing$ and/or from different channels.

During the current data taking period (run 1b) at the Tevatron, one hopes to collect an integrated luminosity of about $100 \mathrm{pb}^{-1}$ per experiment. For the Main Injector Era, integrated luminosities of the order of $1 \mathrm{fb}^{-1}$ are envisioned [58]. The first run with the Main Injector is currently planned for the period of 1998 2003. Through further upgrades of the Tevatron accelerator complex, an additional factor 10 in luminosity may be gained $\left(\mathrm{TeV}^{*}\right)$. The substantial increase in integrated luminosity will make it possible to test the $W W V$ and $Z \gamma V$ vertices with much greater precision than in current experiments. In Fig. 7 we show the 95\% CL limits on anomalous $W W \gamma$ and $Z Z \gamma$ couplings expected for CDF from $W \gamma$ and $Z \gamma$ production at the Tevatron $(\sqrt{s}=2 \mathrm{TeV})$ for $1 \mathrm{fb}^{-1}$ and $10 \mathrm{fb}^{-1}$. Here, and in all subsequent sensitivity plots, we assume that no deviation from the SM prediction is observed in future experiments. To derive bounds on non-standard $W W V$ couplings a dipole form factor is assumed. For the $Z \gamma V$ couplings we use form factor powers 
Table 1: 95\% CL limits on anomalous $W W V, V=\gamma, Z$, and $Z Z \gamma$ couplings from CDF and $\mathrm{D} \varnothing$. Only one of the independent couplings is allowed to deviate from the SM at a time. The bounds obtained for $Z \gamma \gamma$ couplings are very similar to those derived for the $Z Z \gamma$ couplings and are therefore not shown.

\begin{tabular}{|ccc|}
\hline experiment & channel & limit \\
\hline $\mathrm{CDF}$ & $p \bar{p} \rightarrow W^{ \pm} \gamma \rightarrow \ell^{ \pm} \nu \gamma$ & $-2.3<\Delta \kappa_{\gamma}^{0}<2.2$ \\
& $\ell=e, \mu$ & $-0.7<\lambda_{\gamma}^{0}<0.7$ \\
\hline $\mathrm{D} \varnothing$ & $p \bar{p} \rightarrow W^{ \pm} \gamma \rightarrow \ell^{ \pm} \nu \gamma$ & $-1.6<\Delta \kappa_{\gamma}^{0}<1.8$ \\
& $\ell=e, \mu$ & $-0.6<\lambda_{\gamma}^{0}<0.6$ \\
\hline $\mathrm{CDF}$ & $p \bar{p} \rightarrow W^{ \pm} Z \rightarrow \ell^{+} \ell^{-} j j$ & $-8.6<\Delta \kappa_{Z}^{0}<9.0$ \\
& $\ell=e, \mu$ & $-1.7<\lambda_{Z}^{0}<1.7$ \\
\hline $\mathrm{CDF}$ & $p \bar{p} \rightarrow W^{+} W^{-}, W^{ \pm} Z \rightarrow \ell^{ \pm} \nu j j$ & $-1.0<\Delta \kappa_{V}^{0}<1.1$ \\
& $\ell=e, \mu, \kappa_{\gamma}=\kappa_{Z}, \lambda_{\gamma}=\lambda_{Z}$ & $-0.8<\lambda_{V}^{0}<0.8$ \\
\hline $\mathrm{D} \varnothing$ & $p \bar{p} \rightarrow W^{+} W^{-} \rightarrow \ell_{1} \nu_{1} \ell_{2} \nu_{2}$ & $-2.6<\Delta \kappa_{V}^{0}<2.8$ \\
& $\ell_{1,2}=e, \mu, \kappa_{\gamma}=\kappa_{Z}, \lambda_{\gamma}=\lambda_{Z}$ & $-2.2<\lambda_{V}^{0}<2.2$ \\
\hline $\mathrm{CDF}$ & $p \bar{p} \rightarrow Z \gamma \rightarrow \ell^{+} \ell^{-} \gamma$ & $-3.0<h_{30}^{Z}<2.9$ \\
& $\ell=e, \mu, \Lambda_{F F}=0.5 \mathrm{TeV}$ & $-0.7<h_{40}^{Z}<0.7$ \\
\hline $\mathrm{D} \varnothing$ & $p \bar{p} \rightarrow Z \gamma \rightarrow \ell^{+} \ell^{-} \gamma$ & $-1.9<h_{30}^{Z}<1.8$ \\
& $\ell=e, \mu, \Lambda_{F F}=0.5 \mathrm{TeV}$ & $-0.5<h_{40}^{Z}<0.5$ \\
\hline
\end{tabular}

of $n=3\left(h_{3}^{V}\right)$ and $n=4\left(h_{4}^{V}\right)$. The curves shown in Fig. 7 are obtained from a binned likelihood fit of the photon $E_{T}$ distribution. In the $Z \gamma$ case we also show the constraint from unitarity for $\Lambda_{F F}=1.5 \mathrm{TeV}$. The expected experimental limits are calculated for the same value of $\Lambda_{F F}$. The limits on $Z \gamma \gamma$ couplings are very similar to those found for $Z Z \gamma$ couplings and are therefore not shown. Only $W \rightarrow e \nu_{e}$ and $Z \rightarrow e^{+} e^{-}$decays are taken into account in our analysis. Electrons are required to have $|\eta|<3.6$, with at least one electron in the central region of the detector $(|\eta|<$ 1.0). A pseudorapidity cut of $|\eta(\gamma)|<2.4$ is imposed on photons. The acceptances are calculated using the following transverse energy and separation cuts:

$$
\begin{aligned}
& E_{T}(e)>25 \mathrm{GeV}, \quad \quad E_{T}>25 \mathrm{GeV}, \\
& E_{T}(\gamma)>10 \mathrm{GeV}, \quad \Delta R(e, \gamma)>0.7 .
\end{aligned}
$$

In addition, a cut on the transverse $W$ mass of $m_{T}^{W}>50 \mathrm{GeV}$ and a cluster transverse mass cut of $m_{T}\left(e \gamma ; \not \not_{T}\right)>90 \mathrm{GeV}$ were imposed in the $W \gamma$ case. For $Z \gamma$ production, we require $m\left(e^{+} e^{-} \gamma\right)>100 \mathrm{GeV}$ and $m\left(e^{+} e^{-}\right)>70 \mathrm{GeV}$. The efficiencies for electron 
Figure 7: Projected 95\% CL sensitivity limits for a) $W W \gamma$ couplings from $W \gamma$ production and b) $Z Z \gamma$ couplings from $Z \gamma$ production at the Tevatron for integrated luminosities of $1 \mathrm{fb}^{-1}$ and $10 \mathrm{fb}^{-1}$.

and photon identification were taken from the current CDF analysis, as well as the probability for a jet to fake a photon, $\mathcal{P}_{j \rightarrow \gamma}\left(E_{T}\right)$. The systematic uncertainty from the integrated luminosity, parton densities, and higher order QCD corrections was assumed to be 5\%. From Fig. 7a (7b) one observes that the current limits on anomalous gauge boson couplings can be improved by about a factor $5-15$ (10 $100)$ in $W \gamma(Z \gamma)$ production in the Main Injector Era. An additional factor 10 in integrated luminosity leads to roughly a factor 2 improvement in the sensitivities which can be achieved. The maximum form factor scale which can be probed in $Z \gamma$ production with $1 \mathrm{fb}^{-1}\left(10 \mathrm{fb}^{-1}\right)$ is about a factor 2.6 (3) larger than that accessible with the current data. The limit contours shown in Fig. 7 can be improved by about $20-40 \%$ if $W \rightarrow \mu \nu$ and $Z \rightarrow \mu^{+} \mu^{-}$decays are included in the analysis.

The bounds on $Z \gamma V$ couplings could be further improved by analyzing the reaction $p \bar{p} \rightarrow Z \gamma \rightarrow \bar{\nu} \nu \gamma$. Here the signal consists of a single high $p_{T}$ photon accompanied by a large amount of missing transverse energy. Compared to the charged lepton decay mode of the $Z$ boson, the decay $Z \rightarrow \bar{\nu} \nu$ offers potential advantages. Due to the larger $Z \rightarrow \bar{\nu} \nu$ branching ratio, the differential cross section is about a factor 3 larger than that for $q \bar{q} \rightarrow e^{+} e^{-} \gamma$ and $q \bar{q} \rightarrow \mu^{+} \mu^{-} \gamma$ combined. Furthermore, final state bremsstrahlung and timelike virtual photon diagrams do not contribute to the $\bar{\nu} \nu \gamma$ final state. On the other hand, there are several potentially serious background processes which contribute to $p \bar{p} \rightarrow \gamma p_{T}$, but not to the $\ell^{+} \ell^{-} \gamma$ final state. The two most important background processes are prompt photon production, $p \bar{p} \rightarrow \gamma j$, with the jet rapidity outside the range covered by the detector and thus "faking" missing transverse momentum, and two jet production where one of the jets is misidentified as a photon while the other disappears through the 
beam hole. A parton level simulation of the $\gamma j$ and $j j$ backgrounds in $p \bar{p} \rightarrow \gamma p_{T}$ suggests [19] that those backgrounds can be eliminated by requiring a sufficiently large transverse momentum for the photon.

To estimate the sensitivity of $W^{+} W^{-}, W^{ \pm} Z \rightarrow \ell \nu j j$ and $W Z \rightarrow \ell^{+} \ell^{-} j j, \ell=$ $e, \mu$, to non-standard $W W V$ couplings in future Tevatron experiments, we require charged leptons to have $E_{T}>20 \mathrm{GeV}$ and $|\eta(\ell)|<2$, and impose a missing transverse energy cut of $20 \mathrm{GeV}$. The two leading jets are required to have $E_{T}(j)>30 \mathrm{GeV}$ and $60 \mathrm{GeV}<m(j j)<110 \mathrm{GeV}$. Events containing an extra jet with $E_{T}>50 \mathrm{GeV}$ are vetoed in order to suppress the top quark background and to reduce the effect of QCD corrections [52, 59]. To suppress the $W / Z+$ jets background, a cut on the transverse momentum of the jet pair is imposed, similar to the requirement in the current CDF analysis. The value of the $p_{T}(j j)$ cut varies with the integrated luminosity assumed:

$$
\begin{array}{ll}
p_{T}(j j)>150 \mathrm{GeV} & \text { for } \int \mathcal{L} d t=100 \mathrm{pb}^{-1}, \\
p_{T}(j j)>200 \mathrm{GeV} & \text { for } \int \mathcal{L} d t=1 \mathrm{fb}^{-1}, \\
p_{T}(j j)>250 \mathrm{GeV} & \text { for } \int \mathcal{L} d t=10 \mathrm{fb}^{-1} .
\end{array}
$$

The number of signal events expected is calculated using the event generator of Ref. [55]. The trigger and particle identification efficiencies are assumed to be the same as in the current CDF data analysis. To estimate the $t \bar{t}$ and $W / Z+$ jets background, ISAJET and VECBOS [60] are used. The top quark mass is taken to be $m_{t}=170 \mathrm{GeV}$.

Confidence levels are obtained by counting events above the $p_{T}(j j)$ cut. The resulting 95\% CL contours at $\sqrt{s}=1.8 \mathrm{TeV}$ for integrated luminosities of $100 \mathrm{pb}^{-1}$, $1 \mathrm{fb}^{-1}$ and $10 \mathrm{fb}^{-1}$ are shown in Fig. 8a. To calculate the sensitivity limits in Fig. 8a, we have assumed a form factor scale of $\Lambda_{F F}=2 \mathrm{TeV}$ and the effective Lagrangian scenario of Section 2.2 where the $S U(2)_{L} \times U(1)_{Y}$ symmetry is linearly realized with $f_{B}=f_{W}$ ("HISZ scenario" [8]), which reduces the number of independent $W W V$ couplings from five to two. Choosing $\Delta \kappa_{\gamma}$ and $\lambda_{\gamma}$ as independent parameters, the $W W Z$ couplings are then given by [see Eqs. (12) - (14)]:

$$
\begin{aligned}
\Delta g_{1}^{Z} & =\frac{1}{2 \cos ^{2} \theta_{W}} \Delta \kappa_{\gamma}, \\
\Delta \kappa_{Z} & =\frac{1}{2}\left(1-\tan ^{2} \theta_{W}\right) \Delta \kappa_{\gamma} \\
\lambda_{Z} & =\lambda_{\gamma} .
\end{aligned}
$$

The sensitivity limits depend only marginally on the value of $\Lambda_{F F}$ assumed. The bounds obtained in this scenario are compared in Table 2 with those derived for different relations between the $W W V$ couplings. The sensitivity limits found in the HISZ scenario are seen to be representative. If the Tevatron center of mass energy 
Figure 8: Expected 95\% CL sensitivity limits for the $W W V$ couplings in the HISZ scenario [see Eqs. (61) - (63)] a) from $p \bar{p} \rightarrow W W, W Z \rightarrow \ell \nu j j$ and $\ell^{+} \ell^{-} j j$, and b) from $p \bar{p} \rightarrow$ $W^{ \pm} Z \rightarrow \ell_{1}^{ \pm} \nu_{1} \ell_{2}^{+} \ell_{2}^{-}$at the Tevatron.

Table 2: 95\% CL limits on anomalous $W W V, V=\gamma, Z$ from $p \bar{p} \rightarrow W W, W Z \rightarrow \ell \nu j j$ and $\ell^{+} \ell^{-} j j$ at $\sqrt{s}=1.8 \mathrm{TeV}$ for $\int \mathcal{L} d t=1 \mathrm{fb}^{-1}$ and $\int \mathcal{L} d t=10 \mathrm{fb}^{-1}$. Only one coupling at a time is varied, except for the dependencies noted.

\begin{tabular}{|ccc|}
\hline dependent couplings & $\begin{array}{c}\text { limit } \\
\int \mathcal{L} d t=1 \mathrm{fb}^{-1}\end{array}$ & $\begin{array}{c}\text { limit } \\
\int \mathcal{L} d t=10 \mathrm{fb}^{-1}\end{array}$ \\
\hline Eqs. (61) and (62) & $-0.31<\Delta \kappa_{\gamma}^{0}<0.41$ & $-0.17<\Delta \kappa_{\gamma}^{0}<0.24$ \\
$\lambda_{\gamma}=\lambda_{Z}$ & $-0.19<\lambda_{\gamma}^{0}<0.19$ & $-0.10<\lambda_{\gamma}^{0}<0.11$ \\
$\Delta \kappa_{\gamma}=\Delta \kappa_{Z}$ & $-0.23<\Delta \kappa_{V}^{0}<0.29$ & $-0.12<\Delta \kappa_{V}^{0}<0.16$ \\
- & $-0.35<\Delta g_{1}^{Z 0}<0.43$ & $-0.19<\Delta g_{1}^{Z 0}<0.25$ \\
- & $-0.30<\Delta \kappa_{Z}^{0}<0.37$ & $-0.16<\Delta \kappa_{Z}^{0}<0.20$ \\
- & $-0.22<\lambda_{Z}^{0}<0.22$ & $-0.11<\lambda_{Z}^{0}<0.11$ \\
- & $-0.56<\lambda_{\gamma}^{0}<0.56$ & $-0.28<\lambda_{\gamma}^{0}<0.29$ \\
\hline
\end{tabular}


can be increased to $2 \mathrm{TeV}$ the results shown in Fig. 8a and Table 2 improve by a few per cent.

For integrated luminosities $\geq 1 \mathrm{fb}^{-1}, W W$ and $W Z$ production with all leptonic decays can also be used to constrain the $W W V$ vertices. In contrast to the semihadronic $W W, W Z$ production channels, double leptonic $W Z$ decays are relatively background free and thus provide an excellent testing ground for non-standard $W W Z$ couplings. Using a recent calculation of $W^{ \pm} Z$ production which includes NLO QCD corrections [59], sensitivity limits for the $p \bar{p} \rightarrow W^{ \pm} Z \rightarrow \ell_{1}^{ \pm} \nu_{1} \ell_{2}^{+} \ell_{2}^{-}, \ell_{1,2}=e, \mu$, channel were estimated. No full detector simulation was carried out, however, lepton identification cuts of $p_{T}\left(\ell_{1,2}\right)>20 \mathrm{GeV}$ and $\left|\eta\left(\ell_{1,2}\right)\right|<2.5$, and a missing $p_{T}$ cut of $20 \mathrm{GeV}$ have been imposed to roughly simulate detector response. Particle momenta are smeared according to the resolution of the CDF detector. The $95 \%$ CL limit contours for $\sqrt{s}=1.8 \mathrm{TeV}$ and $\Lambda_{F F}=1 \mathrm{TeV}$, obtained from a $\chi^{2}$ fit to the $p_{T}(Z)$ distribution are displayed in Fig. 8b. Here we have again assumed the relations of Eqs. (61) - 63) for $W W \gamma$ and $W W Z$ couplings. If the center of mass energy of the Tevatron can be increased to $2 \mathrm{TeV}$, slightly better limits can be obtained. For $\int \mathcal{L} d t=1 \mathrm{fb}^{-1}$, the small number of $\ell_{1}^{ \pm} \nu_{1} \ell_{2}^{+} \ell_{2}^{-}$events severely limits the sensitivity, and the limits obtained from $W W, W Z \rightarrow \ell \nu j j$ and $\ell^{+} \ell^{-} j j$ are significantly better than those from double leptonic $W Z$ decays for most of the parameter space. For $10 \mathrm{fb}^{-1}$, the non-negligible background starts to negatively influence the semihadronic channels, and double leptonic and $W W, W Z \rightarrow \ell \nu j j$ and $\ell^{+} \ell^{-} j j$ final states yield comparable results. In contrast to double leptonic $W Z$ decays, the $W W \rightarrow \ell_{1} \nu_{1} \ell_{2} \nu_{2}$ final states are plagued by background from $t \bar{t}$ production, and thus were not studied in detail. The contour limits shown in Figs. 7a and 8 depend only marginally on the form factor scale assumed; only the limits on the $Z \gamma V$ couplings are more sensitive to the value of $\Lambda_{F F}$ chosen.

The expected sensitivity bounds from future Tevatron experiments, varying only one of the independent couplings at a time, are summarized in Table 3. Future experiments at the Tevatron can measure $\Delta \kappa_{\gamma}$ with a precision of about $0.1-0.2$. $\lambda_{\gamma}$ can be determined to better than about 0.1 for $\int \mathcal{L} d t \geq 1 \mathrm{fb}^{-1}$. The limits for $Z \gamma V$ couplings are of order $10^{-2}-10^{-3}$.

\subsubsection{Di-boson Production at the $\mathrm{LHC}$}

Since terms proportional to the non-standard $W W V$ and $Z \gamma V$ couplings in the di-boson production amplitudes grow with energy like a power of $\sqrt{\hat{s}} / m_{W}$, one expects [61] that experiments at the LHC $(p p$ collisions at $\sqrt{s}=14 \mathrm{TeV} ; \mathcal{L}=$ $1.7 \cdot 10^{34} \mathrm{~cm}^{-2} \mathrm{~s}^{-1}$ [62]) will be able to improve significantly the limits which can be obtained at the Tevatron. To simulate the sensitivity of $W \gamma$ and $Z \gamma$ production at the LHC to non-standard three vector boson couplings, we use the photon, electron and $\not_{T}$ resolutions of the current ATLAS detector design [63]. Similar results are obtained if CMS 64 specifications are employed. Only $W \rightarrow e \nu_{e}$ and $Z \rightarrow e^{+} e^{-}$ decays are studied. Acceptances are obtained using the following set of cuts:

$$
E_{T}(e)>40 \mathrm{GeV}, \quad \quad \not_{T}>25 \mathrm{GeV},
$$


Table 3: Expected 95\% CL limits on anomalous $W W V, V=\gamma, Z$, and $Z Z \gamma$ couplings from future Tevatron experiments. Only one of the independent couplings is assumed to deviate from the SM at a time. The limits found for $Z \gamma \gamma$ couplings are very similar to those obtained for $h_{3}^{Z}$ and $h_{4}^{Z}$.

\begin{tabular}{|ccc|}
\hline channel & $\begin{array}{c}\text { limit } \\
\int \mathcal{L} d t=1 \mathrm{fb}^{-1}\end{array}$ & $\begin{array}{c}\text { limit } \\
\int \mathcal{L} d t=10 \mathrm{fb}^{-1}\end{array}$ \\
\hline$p \bar{p} \rightarrow W^{ \pm} \gamma \rightarrow e^{ \pm} \nu \gamma$ & $-0.38<\Delta \kappa_{\gamma}^{0}<0.38$ & $-0.21<\Delta \kappa_{\gamma}^{0}<0.21$ \\
$\sqrt{s}=2 \mathrm{TeV}$ & $-0.12<\lambda_{\gamma}^{0}<0.12$ & $-0.057<\lambda_{\gamma}^{0}<0.057$ \\
\hline$p \bar{p} \rightarrow W^{+} W^{-}, W^{ \pm} Z \rightarrow \ell^{ \pm} \nu j j, \ell^{+} \ell^{-} j j$ & $-0.31<\Delta \kappa_{\gamma}^{0}<0.41$ & $-0.17<\Delta \kappa_{\gamma}^{0}<0.24$ \\
$\ell=e, \mu, \mathrm{HISZ}$ scenario & $-0.19<\lambda_{\gamma}^{0}<0.19$ & $-0.10<\lambda_{\gamma}^{0}<0.11$ \\
\hline$p \bar{p} \rightarrow W^{ \pm} Z \rightarrow \ell_{1}^{ \pm} \nu_{1} \ell_{2}^{+} \ell_{2}^{-}$ & $-0.26<\Delta \kappa_{\gamma}^{0}<0.70$ & $-0.09<\Delta \kappa_{\gamma}^{0}<0.32$ \\
$\ell_{1,2}=e, \mu, \mathrm{HISZ}$ scenario & $-0.24<\lambda_{\gamma}^{0}<0.32$ & $-0.10<\lambda_{\gamma}^{0}<0.13$ \\
\hline$p \bar{p} \rightarrow Z \gamma \rightarrow e^{+} e^{-} \gamma$ & $-0.105<h_{30}^{Z}<0.105$ & $-0.044<h_{30}^{Z}<0.044$ \\
$\sqrt{s}=2 \mathrm{TeV}, \Lambda_{F F}=1.5 \mathrm{TeV}$ & $-0.0064<h_{40}^{Z}<0.0064$ & $-0.0025<h_{40}^{Z}<0.0025$ \\
\hline
\end{tabular}

$$
\begin{array}{rlrl}
E_{T}(\gamma) & >25 \mathrm{GeV}, & & \Delta R(e, \gamma)>0.7, \\
m_{T}^{W}>50 \mathrm{GeV}, & & m_{T}\left(e \gamma ; \dot{k}_{T}\right)>90 \mathrm{GeV},
\end{array}
$$

and

$$
m\left(e^{+} e^{-}\right)>70 \mathrm{GeV}, \quad m\left(e^{+} e^{-} \gamma\right)>110 \mathrm{GeV} .
$$

Since most of the sensitivity to anomalous couplings originates from the high $E_{T}$ tail, the limits which can be obtained change only very little if the $E_{T}(\gamma)\left(\not_{T}\right)$ cut is raised to $50-100 \mathrm{GeV}(40-50 \mathrm{GeV})$. For the electron and photon identification efficiencies, the values obtained in the current CDF analysis were used. The systematic uncertainty from the integrated luminosity, parton densities, and higher order QCD corrections was varied between 5\% and 10\%. NLO QCD corrections are known to be large at LHC energies, and significantly reduce the sensitivity to anomalous couplings, unless a jet veto is imposed [65]. All jets in $W \gamma$ and $Z \gamma$ events with a transverse energy larger than $50 \mathrm{GeV}$ were therefore vetoed. This cut also helps to reduce to an acceptable level the background from $p p \rightarrow \bar{t} t \gamma \rightarrow W \gamma+X$ and, together with the photon and lepton isolation cuts, the $p p \rightarrow \bar{b} b \gamma$ background [66, 67].

The $95 \%$ CL limit contours from a binned likelihood fit of the photon $E_{T}$ distribution for an integrated luminosity of $100 \mathrm{fb}^{-1}$ are shown in Fig. 9. To obtain the results shown in this figure, we have assumed a systematic uncertainty of $5 \%$. Almost identical curves are obtained if the systematic uncertainty is increased to $10 \%$. In contrast to the sensitivities obtained at Tevatron energies, the limits on 
Figure 9: $95 \% C L$ sensitivity limits for a) $W W \gamma$ couplings from $W \gamma$ production and b) $Z Z \gamma$ couplings from $Z \gamma$ production at the LHC. Results are displayed for an integrated luminosity of $100 \mathrm{fb}^{-1}$ and two different form factor scales.

$W W \gamma$ couplings found for $p p$ collisions at $\sqrt{s}=14 \mathrm{TeV}$ depend non-negligibly on the form factor scale. The bounds on $\Delta \kappa_{\gamma}^{0}\left(\lambda_{\gamma}^{0}\right)$ are about a factor 3 to $4(\sim 10)$ better than those possible at the Tevatron with $10 \mathrm{fb}^{-1}$. The limits on $Z \gamma V$ couplings can be improved by a factor $\sim 10\left(h_{30}^{V}\right)$ to $\sim 30\left(h_{40}^{V}\right)$ for $\Lambda_{F F}=1.5 \mathrm{TeV}$. The $95 \% \mathrm{CL}$ limit contours for the $Z \gamma \gamma$ couplings are almost identical to those found for $h_{30}^{Z}$ and $h_{40}^{Z}$ and are therefore not shown in Fig. 9b. The limits obtained for $Z \gamma V$ couplings depend very strongly on the value of $\Lambda_{F F}$ assumed. Increasing the form factor scale from $1.5 \mathrm{TeV}$ to $3 \mathrm{TeV}$, the limits improve by a factor 5 to 10 . The results shown in Fig. 9 can be improved by about $20-40 \%$ if $W \rightarrow \mu \nu$ and $Z \rightarrow \mu^{+} \mu^{-}$decays are included in the analysis. The limits on anomalous $Z \gamma V$ couplings could be further strengthened if the $Z \rightarrow \bar{\nu} \nu$ decay can be utilized.

Using the NLO calculation of Ref. [59], sensitivity limits for the reaction $p p \rightarrow$ $W^{ \pm} Z \rightarrow \ell_{1}^{ \pm} \nu_{1} \ell_{2}^{+} \ell_{2}^{-}$were estimated by performing a $\chi^{2}$ fit to the $p_{T}(Z)$ distribution. No complete detector simulation was carried out, however, a transverse momentum cut of $25 \mathrm{GeV}$ and a rapidity cut of $\left|\eta\left(\ell_{1,2}\right)\right|<2.5, \ell_{1,2}=e, \mu$, were imposed on the charged leptons, together with a missing transverse energy cut of $50 \mathrm{GeV}$. The relatively large $\not_{T}$ cut was chosen to reduce backgrounds e.g from event pileup which at LHC luminosities may result in a non-negligible amount of "fake" missing transverse energy [68], and from processes such as $p p \rightarrow Z b \bar{b} \rightarrow \ell_{1} \nu_{1} \ell_{2}^{+} \ell_{2}^{-}+X$. The large $\not_{T}$ cut has only very little impact on the sensitivity limits which can be achieved. In addition, leptons of the same charge are required to be separated by $\Delta R>0.4$. To reduce the effect of QCD corrections, and the $p p \rightarrow t \bar{t} \rightarrow \ell_{1} \ell_{2} \ell_{2}+X$ 63 and $p p \rightarrow \bar{t} t Z$ [66] backgrounds on the sensitivity limits, jets with $p_{T}(j)>50 \mathrm{GeV}$ and $|\eta(j)|<2.5$ are vetoed. Particle momenta are smeared according to the resolution 
Figure 10: $95 \% C L$ sensitivity limits from $W^{ \pm} Z \rightarrow \ell_{1}^{ \pm} \nu_{1} \ell_{2}^{+} \ell_{2}^{-}$at the $L H C$ a) in the HISZ scenario and b) if only $\Delta \kappa_{Z}$ and $\lambda_{Z}$ are allowed to deviate from the $S M$.

expected for the ATLAS detector [63]. A 50\% normalization uncertainty of the SM $p_{T}(Z)$ distribution was taken into account in the derivation of the $95 \% \mathrm{CL}$ limit contours, which are shown in Fig. 10 for $\int \mathcal{L} d t=100 \mathrm{fb}^{-1}$ and two choices for the form factor scale. In Fig. 10a we show 95\% CL limits for the HISZ scenario [see Eqs. (61) - 63)]. Figure 10b displays sensitivity bounds for the case where only $\Delta \kappa_{Z}$ and $\lambda_{Z}$ are varied.

At the LHC, the $t \bar{t}$ production rate for top quark masses in the range from $150 \mathrm{GeV}$ to $200 \mathrm{GeV}$ is about a factor 10 to 30 larger than the $p p \rightarrow W^{+} W^{-}$ cross section [69]. Unless the top quark background can be reduced very efficiently, one does not expect that $W^{+} W^{-}$and semihadronic $W Z$ production yield limits on anomalous $W W V$ couplings which can compete with those obtained from $p p \rightarrow W \gamma$ and double leptonic $W Z$ decays.

Table 4 compares the sensitivities which can be achieved in $W \gamma, W Z$ and $Z \gamma$ production at the LHC with $100 \mathrm{fb}^{-1}$. If the integrated luminosity is reduced by a factor 10, the bounds listed in Table 4 are weakened by about a factor $2 . \Delta \kappa_{V}$ and $\lambda_{V}$ in general can be probed to better than 0.1 and 0.01 at the LHC, respectively. The limits which are obtained in the HISZ scenario for $\Delta \kappa_{\gamma}$ from $W^{ \pm} Z \rightarrow \ell_{1}^{ \pm} \nu_{1} \ell_{2}^{+} \ell_{2}^{-}$ are $\mathcal{O}\left(10^{-2}\right)$ and thus much stronger than those from $W \gamma$ production. This is due to the relation between $\Delta g_{1}^{Z}$ and $\Delta \kappa_{\gamma}$ in the HISZ scenario [see Eq. (61)], and the fact that the terms proportional to $\Delta g_{1}^{Z}$ in $W Z$ production grow like $\hat{s} / m_{W}^{2}$ with energy, whereas the terms proportional to $\Delta \kappa_{\gamma}$ in $W \gamma$ production only increase like $\sqrt{\hat{s}} / m_{W}$ at most. Varying the form factor scale from $3 \mathrm{TeV}$ to $10 \mathrm{TeV}$, the limits on $W W V$ couplings improve by about $30 \%$. For $\Lambda_{F F}<3 \mathrm{TeV}$, the bounds deteriorate rather quickly; for $\Lambda_{F F}=1 \mathrm{TeV}$ they are a factor $2-5$ weaker than those found for $\Lambda_{F F}=3 \mathrm{TeV}$. As mentioned before, the sensitivities obtained for $Z \gamma V$ couplings 
Table 4: Expected 95\% CL limits on anomalous $W W V, V=\gamma, Z$, and $Z Z \gamma$ couplings from experiments at the LHC ( $p p$ collisions at $\left.\sqrt{s}=14 \mathrm{TeV} ; \int \mathcal{L} d t=100 \mathrm{fb}^{-1}\right)$. Only one of the independent couplings is assumed to deviate from the SM at a time. The limits obtained for $Z \gamma \gamma$ couplings almost coincide with those found for $h_{3}^{Z}$ and $h_{4}^{Z}$.

\begin{tabular}{|ccc|}
\hline channel & $\begin{array}{c}\text { limit } \\
\Lambda_{F F}=3 \mathrm{TeV}\end{array}$ & $\begin{array}{c}\text { limit } \\
\Lambda_{F F}=10 \mathrm{TeV}\end{array}$ \\
\hline$p p \rightarrow W^{ \pm} \gamma \rightarrow e^{ \pm} \nu \gamma$ & $-0.080<\Delta \kappa_{\gamma}^{0}<0.080$ & $-0.065<\Delta \kappa_{\gamma}^{0}<0.065$ \\
& $-0.0057<\lambda_{\gamma}^{0}<0.0057$ & $-0.0032<\lambda_{\gamma}^{0}<0.0032$ \\
\hline$p p \rightarrow W^{ \pm} Z \rightarrow \ell_{1}^{ \pm} \nu_{1} \ell_{2}^{+} \ell_{2}^{-}$ & $-0.0060<\Delta \kappa_{\gamma}^{0}<0.0097$ & $-0.0043<\Delta \kappa_{\gamma}^{0}<0.0086$ \\
$\ell_{1,2}=e, \mu, \mathrm{HISZ}$ scenario & $-0.0053<\lambda_{\gamma}^{0}<0.0067$ & $-0.0043<\lambda_{\gamma}^{0}<0.0038$ \\
\hline$p p \rightarrow W^{ \pm} Z \rightarrow \ell_{1}^{ \pm} \nu_{1} \ell_{2}^{+} \ell_{2}^{-}$ & $-0.064<\Delta \kappa_{Z}^{0}<0.107$ & $-0.050<\Delta \kappa_{Z}^{0}<0.078$ \\
$\ell_{1,2}=e, \mu, \Delta g_{1}^{Z}=0$ & $-0.0076<\lambda_{Z}^{0}<0.0075$ & $-0.0043<\lambda_{Z}^{0}<0.0038$ \\
\hline channel & $\operatorname{limit}$ & $\operatorname{limit}$ \\
& $\Lambda_{F F}=1.5 \mathrm{TeV}$ & $\Lambda_{F F}=3 \mathrm{TeV}$ \\
\hline$p p \rightarrow Z \gamma \rightarrow e^{+} e^{-} \gamma$ & $-0.0051<h_{30}^{Z}<0.0051$ & $-0.0013<h_{30}^{Z}<0.0013$ \\
& $-9.2 \cdot 10^{-5}<h_{40}^{Z}<9.2 \cdot 10^{-5}$ & $-6.8 \cdot 10^{-6}<h_{40}^{Z}<6.8 \cdot 10^{-6}$ \\
\hline
\end{tabular}


depend even more strongly on the form factor scale. A maximum scale of $\sim 10 \mathrm{TeV}$ can be probed in $W \gamma$ and $W Z$ production, whereas scales up to $6 \mathrm{TeV}$ are accessible in $Z \gamma$ production at the LHC. The limits from $W \gamma$ and $W Z$ production listed in Table 4 are consistent with those found in Ref. [63].

\subsubsection{Amplitude Zeros and Rapidity Correlations in $W \gamma$ and $W Z$ Production}

$W \gamma$ and $W Z$ production in hadronic collisions are of special interest due to the presence of amplitude zeros. It is well known that all SM helicity amplitudes of the parton-level subprocess $q_{1} \bar{q}_{2} \rightarrow W^{ \pm} \gamma$ vanish for [30]

$$
\cos \theta=\frac{Q_{1}+Q_{2}}{Q_{1}-Q_{2}}
$$

where $\theta$ is the scattering angle of the $W$-boson with respect to the quark $\left(q_{1}\right)$ direction in the $W \gamma$ rest frame, and $Q_{i}(i=1,2)$ are the quark charges in units of the proton electric charge $e$. This zero is a consequence of the factorizability $[70$ of the amplitudes in gauge theories into one factor which contains the gauge coupling dependence and another which contains spin information. Although the factorization holds for any four-particle Born-level amplitude in which one or more of the four particles is a gauge-field quantum, the amplitudes for most processes may not necessarily develop a kinematical zero in the physical region. The amplitude zero in the $W^{ \pm} \gamma$ process has been further shown to correspond to the absence of dipole radiation by colliding particles with the same charge-to-mass ratio [71, a realization of classical radiation interference.

Recently, it was found [31] that the SM amplitude of the process $q_{1} \bar{q}_{2} \rightarrow W^{ \pm} Z$ also exhibits an approximate zero at high energies. The $( \pm, \mp)$ amplitudes $\mathcal{M}( \pm, \mp)$ vanish for

$$
\frac{g_{-}^{q_{1}}}{\hat{u}}+\frac{g_{-}^{q_{2}}}{\hat{t}}=0
$$

where $g_{-}^{q_{i}}$ is the coupling of the $Z$ boson to left-handed quarks, and $\hat{u}$ and $\hat{t}$ are Mandelstam variables in the parton center of mass frame. For $\hat{s} \gg m_{Z}^{2}$, the zero in the $( \pm, \mp)$ amplitudes is located at $\cos \theta_{0}=\left(g_{-}^{q_{1}}+g_{-}^{q_{2}}\right) /\left(g_{-}^{q_{1}}-g_{-}^{q_{2}}\right)$, or

$$
\cos \theta_{0} \simeq \begin{cases}+\frac{1}{3} \tan ^{2} \theta_{\mathrm{w}} \simeq+0.1 \text { for } d \bar{u} \rightarrow W^{-} Z, \\ -\frac{1}{3} \tan ^{2} \theta_{\mathrm{w}} \simeq-0.1 \text { for } u \bar{d} \rightarrow W^{+} Z .\end{cases}
$$

The existence of the zero in $\mathcal{M}( \pm, \mp)$ at $\cos \theta_{0}$ is a direct consequence of the contributing Feynman diagrams and the left-handed coupling of the $W$-boson to fermions.

At high energies, strong cancellations occur, and, besides $\mathcal{M}( \pm, \mp)$, only the $(0,0)$ amplitude remains non-zero. The combined effect of the zero in $\mathcal{M}( \pm, \mp)$ and the gauge cancellations at high energies in the remaining helicity amplitudes results in an approximate zero for the $q_{1} \bar{q}_{2} \rightarrow W^{ \pm} Z$ differential cross section at $\cos \theta \approx \cos \theta_{0}$.

Non-standard $W W V$ couplings in general destroy the amplitude zeros in $W \gamma$ and $W Z$ production. Searching for the amplitude zeros thus provides an additional test of the gauge theory nature of the SM. 
Unfortunately, the radiation zero in $q_{1} \bar{q}_{2} \rightarrow W \gamma \rightarrow \ell \nu \gamma$ and the approximate amplitude zero in $q_{1} \bar{q}_{2} \rightarrow W Z \rightarrow \ell_{1} \nu_{1} \ell_{2}^{+} \ell_{2}^{-}$are not easy to observe in the $\cos \theta$ distribution in $p p$ or $p \bar{p}$ collider experiments. Structure function effects transform the zero in the $W \gamma$ case into a dip in the $\cos \theta$ distribution. The approximate zero in $W Z$ production is only slightly affected by structure function effects. Higher order QCD corrections [72] and finite $W$ width effects [73] tend to fill in the dip. In $W \gamma$ production photon radiation from the final state lepton line also diminishes the significance of the effect.

The main complication in the extraction of the $\cos \theta$ distribution, however, originates from the finite resolution of the detector and ambiguities in reconstructing the parton center of mass frame. The ambiguities are associated with the nonobservation of the neutrino arising from $W$ decay. Identifying the missing transverse momentum with the transverse momentum of the neutrino of a given $W \gamma$ or $W Z$ event, the unobservable longitudinal neutrino momentum, $p_{L}(\nu)$, and thus the parton center of mass frame, can be reconstructed by imposing the constraint that the neutrino and charged lepton four momenta combine to form the $W$ rest mass 74 . The resulting quadratic equation, in general, has two solutions. In the approximation of a zero $W$ decay width, one of the two solutions coincides with the true $p_{L}(\nu)$. On an event to event basis, however, it is impossible to tell which of the two solutions is the correct one. This ambiguity considerably smears out the dip caused by the amplitude zeros.

Instead of trying to reconstruct the parton center of mass frame and measure the $\cos \theta$ or the equivalent rapidity distribution in the center of mass frame, one can study rapidity correlations between the observable final state particles in the laboratory frame [75]. Knowledge of the neutrino longitudinal momentum is not required in determining the rapidity correlations. Event mis-reconstruction problems originating from the two possible solutions for $p_{L}(\nu)$ are thus automatically avoided.

In $2 \rightarrow 2$ reactions differences of rapidities are invariant under boosts. One therefore expects that the rapidity difference distributions $d \sigma / d \Delta y(V, W), V=\gamma, Z$, where $\Delta y(V, W)=y(V)-y(W)$ and $y(W), y(V)$ are the rapidities in the laboratory frame, exhibit a dip signaling the SM amplitude zeros [75]. In $W^{ \pm} \gamma$ production, the dominant $W$ helicity is $\lambda_{W}= \pm 1$ [76], implying that the charged lepton, $\ell=e, \mu$, from $W \rightarrow \ell \nu$ tends to be emitted in the direction of the parent $W$, and thus reflects most of its kinematic properties. As a result, the dip signaling the SM radiation zero should manifest itself in the $\Delta y(\gamma, \ell)=y(\gamma)-y(\ell)$ distribution.

The SM $\Delta y(\gamma, \ell)$ differential cross section for $p \bar{p} \rightarrow \ell^{+} p_{T} \gamma$ at the Tevatron is shown in Fig. 11a. To simulate detector response, transverse momentum cuts of $p_{T}(\gamma)>5 \mathrm{GeV}, p_{T}(\ell)>20 \mathrm{GeV}$ and $p_{T}>20 \mathrm{GeV}$, rapidity cuts of $|y(\gamma)|<3$ and $|y(\ell)|<3.5$, a cluster transverse mass cut of $m_{T}\left(\ell \gamma ; p_{T}\right)>90 \mathrm{GeV}$ and a lepton photon separation cut of $\Delta R(\gamma, \ell)>0.7$ have been imposed. The SM radiation zero is seen to lead to a strong dip in the $\Delta y(\gamma, \ell)$ distribution at $\Delta y(\gamma, \ell) \approx-0.3$. Next-to-leading QCD corrections do not seriously affect the significance of the dip. However, a sufficient rapidity coverage is essential to observe the radiation zero in the $\Delta y(\gamma, \ell)$ distribution [75]. 
Figure 11: Rapidity difference distributions in the $S M$ at the Tevatron. a) The photon lepton rapidity difference spectrum in $p \bar{p} \rightarrow \ell^{+} \not p_{T} \gamma$. b) The $y(Z)-y\left(\ell_{1}^{+}\right)$and $y\left(\ell_{2}^{+}\right)-y\left(\ell_{1}^{+}\right)$ distributions in $p \bar{p} \rightarrow W^{+} Z$.

In contrast to the situation in $W \gamma$ production, none of the $W$ helicities dominates in $W Z$ production [76]. The charged lepton originating from the $W$ decay, $W \rightarrow \ell_{1} \nu_{1}$, thus only partly reflects the kinematical properties of the parent $W$ boson. As a result, a significant part of the correlation present in the $y(Z)-y(W)$ spectrum [77] is lost, and only a slight dip survives in the $y(Z)-y\left(\ell_{1}\right)$ distribution, which is shown for the $W^{+} Z$ case in Fig. 11b. The dip in the SM $y(Z)-y\left(\ell_{1}\right)$ distribution will thus be more difficult to observe experimentally than that in the $y(\gamma)-y(\ell)$ distribution in $W \gamma$ production. Next-to-leading order QCD corrections have only little impact on the shape of the $y(Z)-y\left(\ell_{1}\right)$ distribution [59]. The cuts used in Fig. 11b are the same as those in Fig. 3a except for the lepton rapidity cut which has been replaced by $\left|y\left(\ell_{1,2}\right)\right|<2.5$.

Although the $Z$ boson rapidity, $y(Z)$, can readily be reconstructed from the four momenta of the lepton pair $\ell_{2}^{+} \ell_{2}^{-}$originating from the $Z$ decay, it would be easier experimentally to directly study the rapidity correlations between the charged leptons originating from the $Z \rightarrow \ell_{2}^{+} \ell_{2}^{-}$and $W \rightarrow \ell_{1} \nu_{1}$ decays. The dotted line in Fig. 11b shows the $y\left(\ell_{2}^{+}\right)-y\left(\ell_{1}^{+}\right)$distribution for $W^{+} Z$ production at the Tevatron. The $y\left(\ell_{2}^{-}\right)-y\left(\ell_{1}^{+}\right)$spectrum almost coincides with the $y\left(\ell_{2}^{+}\right)-y\left(\ell_{1}^{+}\right)$distribution. Since also none of the $Z$ boson helicities dominates [76] in $q_{1} \bar{q}_{2} \rightarrow W Z$, the rapidities of the leptons from $W$ and $Z$ decays are almost completely uncorrelated, and essentially no 
Figure 12: Rapidity difference distributions in the $S M$ at the LHC. a) The photon lepton rapidity difference spectrum in $p p \rightarrow \ell^{+} \not p_{T} \gamma$. b) The $y(Z)-y\left(\ell_{1}^{+}\right)$distribution in $p p \rightarrow$ $W^{+} Z$.

trace of the dip signaling the approximate amplitude zero is left in the $y\left(\ell_{2}^{+}\right)-y\left(\ell_{1}^{+}\right)$ distribution.

In $p p$ collisions, the dip signaling the amplitude zeros is shifted to $\Delta y=0$. Because of the large $q g$ luminosity, the inclusive QCD corrections are very large for $W \gamma$ and $W Z$ production [59, 65]. At the LHC, they enhance the cross section by a factor $2-3$. The rapidity difference distributions for $W^{+} \gamma$ and $W^{+} Z$ production in the SM for $p p$ collisions at $\sqrt{s}=14 \mathrm{TeV}$ are shown in Fig. 12. Here we have imposed the following lepton and photon detection cuts:

$$
\begin{aligned}
p_{T}(\gamma)>100 \mathrm{GeV}, & |\eta(\gamma)|<2.5, \\
p_{T}(\ell)>25 \mathrm{GeV}, & |\eta(\ell)|<3, \\
p_{T}>50 \mathrm{GeV}, & \Delta R(\gamma, \ell)>0.7
\end{aligned}
$$

together with a $\Delta R(\ell, \ell)>0.4$ requirement on leptons of the same charge in $W Z$ production. The inclusive NLO QCD corrections are seen to considerably obscure the amplitude zeros. The bulk of the corrections at LHC energies originates from quark gluon fusion and the kinematical region where e.g. the photon or $Z$ boson is produced at large $p_{T}$ and recoils against a quark, which radiates a soft $W$ boson which is almost collinear to the quark. Events which originate from this phase space region usually contain a high $p_{T}$ jet. A jet veto therefore helps to reduce 
the QCD corrections, as demonstrated by the dotted lines in Fig. 12. Here a jet is defined as a quark or gluon with $p_{T}(j)>50 \mathrm{GeV}$ and $|\eta(j)|<3$. Nevertheless, the remaining QCD corrections still substantially reduce the visibility of the radiation zero in $W \gamma$ production at the LHC. In $p p \rightarrow W Z$, the difference in significance of the dip between the LO and the NLO 0-jet $\Delta y\left(Z, \ell_{1}\right)$ distribution is quite small.

Given a sufficiently large integrated luminosity, experiments at the Tevatron studying lepton photon rapidity correlations offer a much better chance to observe the SM radiation zero in $W \gamma$ production than experiments at the LHC. Searching for the approximate amplitude zero in $W Z$ production will be difficult at the Tevatron as well as the LHC.

Indirectly, the radiation zero can also be observed in the $Z \gamma$ to $W \gamma$ cross section ratio [78]. Many theoretical and experimental uncertainties at least partially cancel in the cross section ratio. On the other hand, in searching for the effects of the SM radiation zero in the $Z \gamma$ to $W \gamma$ cross section ratio, one has to assume that the $\mathrm{SM}$ is valid for $Z \gamma$ production. Similarly, the $Z Z$ to $W Z$ cross section ratio reflects the approximate amplitude zero in $W Z$ production, whereas the ratio of $W Z$ to $W \gamma$ cross sections measures the relative strength of the zeros in $W Z$ and $W \gamma$ production [59].

\subsection{Probing $W W V$ and $Z \gamma V$ Couplings in $e^{+} e^{-}$Collider Experiments}

\subsubsection{Single Photon Production at LEP}

In $e^{+} e^{-}$collisions at center of mass energies near the $Z$ boson mass, anomalous $Z \gamma V$ couplings would affect the production of $f \bar{f} \gamma$ final states. At LEP energies, the production of single photons is the process which is most sensitive to anomalous $Z Z \gamma$ couplings, due to the large branching ratio for $Z \rightarrow \nu \bar{\nu}$ decays and the absence of background from final state radiation or final state $\pi^{0}$ 's misidentified as photons. In order to probe $Z \gamma \gamma$ couplings one has to study $\ell^{+} \ell^{-} \gamma$ or $j j \gamma$ final states.

The L3 Collaboration has searched for anomalous $Z Z \gamma$ couplings in single photon events in the data collected in $1991-93$ [79]. Non-standard $Z Z \gamma$ couplings mostly affect the production of energetic single photon events whereas the photon energy spectrum in the SM process $e^{+} e^{-} \rightarrow \bar{\nu} \nu \gamma$ is peaked at low energies. Therefore, a cluster in the BGO electromagnetic calorimeter with energy greater than half the beam energy was required. In order to further reduce the SM contribution and to eliminate the background from QED events in which all final state particles except the photon escape undetected down the beampipe or into a detector crack, it was required that the polar angle of the most energetic cluster lies between 20 and 160 degrees (excluding the ranges between 34.5 and 44.5, and 135.5 and 145 degrees due to gaps between the forward and barrel BGO calorimeters). To suppress

the background from cosmic events, the transverse shape of the BGO cluster was required to be consistent with a photon originating from the interaction point. Apart from the energetic BGO cluster, all other activity in the detector had to be consistent with noise. In terms of equivalent integrated luminosity at the peak of the $Z$ resonance, the data sample corresponds to $50.8 \mathrm{pb}^{-1}$. One event was selected. The number of events expected in the SM is 1.2 . 
Figure 13: Present limits on anomalous $Z Z \gamma$ couplings from $Z \rightarrow \bar{\nu} \nu \gamma$, and from $Z \gamma$ production at the Tevatron.

Since the level of energetic single photon production is consistent with what is expected in the SM, upper limits on the $Z Z \gamma$ couplings can be derived. To extract limits, a modified version of the event generator of Ref. [19] was used. Events were generated for various combinations of $Z Z \gamma$ couplings, and passed through the detector simulation and analysis procedure. Figure 13 shows the 95\% CL upper limits on $h_{30}^{Z}$ and $h_{40}^{Z}$ for a form factor scale of $\Lambda_{F F}=500 \mathrm{GeV}$. Also shown are the current limits from D $\varnothing$ and CDF. Table 5 summarizes the numerical values, if only one of the couplings deviates from the SM at a time. The limits obtained from $Z \rightarrow \bar{\nu} \nu \gamma$ on $h_{30}^{Z}$ are significantly better than those found from $Z \gamma$ production at the Tevatron. On the other hand, because of the larger center of mass energy and the strong increase of the terms proportional to $h_{4}^{Z}$ in the helicity amplitudes, hadron collider experiments give much better bounds on $h_{40}^{Z}$ than single photon production at LEP. LEP and Tevatron experiments thus yield complementary information on $Z Z \gamma$ couplings. LEP will discontinue to run on the $Z$ peak in 1996 . Final integrated statistics are expected to increase by perhaps a factor 3 over that used in the current analysis. Consequently, the present limits on $h_{30}^{Z}$ and $h_{40}^{Z}$ from $Z \rightarrow \bar{\nu} \nu \gamma$ are expected to improve by not more than about a factor 2 in the future. In contrast to the limits obtained from hadron collider experiments, the sensitivity bounds derived from $Z \rightarrow \bar{\nu} \nu \gamma$ only marginally depend on the form factor scale.

An analysis of $\ell^{+} \ell^{-} \gamma$ final states is in progress.

\subsection{2 $W^{+} W^{-}$and $Z \gamma$ Production at LEP II}

$W$ pair production and $Z \gamma$ production at LEP II $(\sqrt{s}=176-190 \mathrm{GeV})$ offer ideal possibilities to probe $W W V$ [3, 80, 81] and $Z \gamma V$ [82 couplings. In contrast to $p p, p \bar{p} \rightarrow W^{+} W^{-} \rightarrow \ell \nu j j$, the reaction $e^{+} e^{-} \rightarrow W^{+} W^{-} \rightarrow \ell \nu j j$ is not plagued by 
Table 5: 95\% CL limits on anomalous $Z \gamma V, V=\gamma, Z$, couplings from L3, CDF and D $\varnothing$. Only one of the independent couplings is allowed to deviate from the SM at a time. The form factor scale is chosen to be $\Lambda_{F F}=500 \mathrm{GeV}$.

\begin{tabular}{|ccc|}
\hline experiment & channel & limit \\
\hline L3 & $e^{+} e^{-} \rightarrow Z \rightarrow \bar{\nu} \nu \gamma$ & $-0.85<h_{30}^{Z}<0.85$ \\
& & $-2.32<h_{40}^{Z}<2.32$ \\
\hline CDF & $p \bar{p} \rightarrow Z \gamma \rightarrow \ell^{+} \ell^{-} \gamma$ & $-3.0<h_{30}^{Z}<2.9$ \\
& $\ell=e, \mu$ & $-0.7<h_{40}^{Z}<0.7$ \\
\hline DØ & $p \bar{p} \rightarrow Z \gamma \rightarrow \ell^{+} \ell^{-} \gamma$ & $-1.9<h_{30}^{Z}<1.8$ \\
& $\ell=e, \mu$ & $-0.5<h_{40}^{Z}<0.5$ \\
\hline
\end{tabular}

large backgrounds. Furthermore, the reconstruction of the leptonically decaying $W$ boson is easier than in hadronic collisions, where the longitudinal momentum of the neutrino can be reconstructed only with a twofold ambiguity. At hadron colliders, limits on non-standard couplings are derived from distributions such as the transverse momentum distribution of one of the vector bosons which make use of the high energy behaviour of the anomalous contributions to the helicity amplitudes. At LEP II, on the other hand, angular distributions are more useful. Different anomalous couplings contribute to different helicity amplitudes and therefore affect the angular distributions in a characteristic way (see Ref. [3]).

In $W^{+} W^{-}$production, 5 angles are available from each event. These are the $W$ production angle, $\Theta_{W}$, and the angles of the $W^{ \pm} \rightarrow f \bar{f}^{\prime}$ decay products in the $W^{ \pm}$ rest frames, $\theta_{ \pm}$and $\phi_{ \pm}$. In the extraction of these angles, two problems have to be faced: First, the imperfect detection of $W$ decay products gives rise to uncertainties in the reconstructed directions of the $W$ 's and their decay products; second, in the case of hadronic $W$ decays, the absence of a readily recognizable quark tag implies that the $W$ decay angles can only be determined with a two-fold ambiguity from the data, resulting in symmetrized angular distributions. Complete information for a $W^{+} W^{-}$event is only available if it is possible to distinguish the $W^{+}$and $W^{-}$ direction.

Of the three final states available in $W$ pair production, $\ell_{1} \nu_{1} \ell_{2} \nu_{2}, \ell \nu j j, \ell=$ $e, \mu$, and $j j j j$, we have only studied the $\ell \nu j j$ channel. The purely leptonic channel is plagued by a small branching ratio $(\approx 4.7 \%)$ and by reconstruction problems due to the presence of two neutrinos. In the $j j j j$ final state it is difficult to discriminate the $W^{+}$and $W^{-}$decay products. Due to the resulting ambiguities in $\Theta_{W}$ and the $W^{ \pm}$ decay angles, the sensitivity bounds which can be achieved from the 4-jet final state are a factor $1.5-2$ weaker than those found from analyzing the $\ell \nu j j$ state [80]. In the $\ell \nu j j$ channel, on the other hand, the identification of the charged lepton allows 
the $W^{+}$and $W^{-}$decays to be distinguished unambiguously.

Events in the $\ell \nu j j$ channel were selected from simulated Monte Carlo data at $\sqrt{s}=176 \mathrm{GeV}$ and $\sqrt{s}=190 \mathrm{GeV}$ using the event generator of Ref. [3]. Initial state radiation and detector smearing, using the L3 specifications are taken into account in the simulations. The following cuts were imposed:

- Number of calorimetric clusters $>16$. This requirement eliminates almost all $W W \rightarrow \ell_{1} \nu_{1} \ell_{2} \nu_{2}, \ell_{1,2}=e, \mu$ events. It also helps to suppress the $W W \rightarrow \tau \nu_{\tau} \ell \nu$, $\ell=e, \mu$ and $W W \rightarrow \tau \nu_{\tau} \tau \nu_{\tau}$ channels where at least one of the $\tau$ leptons decays hadronically. Furthermore it provides some rejection of $e^{+} e^{-} \rightarrow \gamma \gamma$ and $e^{+} e^{-} \rightarrow \tau^{+} \tau^{-}(\gamma)$ events.

- A visible energy $E_{v i s}>80 \mathrm{GeV}$. This cut mainly reduces the background from $e^{+} e^{-} \rightarrow \gamma \gamma$ and $e^{+} e^{-} \rightarrow \tau^{+} \tau^{-}(\gamma)$, removes signal events which are poorly reconstructed, and further suppresses $W W \rightarrow \tau \nu_{\tau} \ell \nu, \ell=e, \mu$ and $W W \rightarrow \tau \nu_{\tau} \tau \nu_{\tau}$ events where at least one of the $\tau$ leptons decays hadronically.

- $\not_{T} / E_{v i s}>0.1$. It reduces the $W W \rightarrow j j j j$ and $Z / \gamma^{*}(\gamma) \rightarrow j j(\gamma)$ backgrounds. Here, " $(\gamma)$ " denotes a photon from initial state radiation.

- The momentum of the most energetic lepton, positively identified as an electron or muon, is $p_{\max }>20 \mathrm{GeV}$. This cut provides most of the suppression of the $W W \rightarrow \tau \nu_{\tau} j j$ and $j j j j$, and $Z / \gamma^{*} \rightarrow j j(\gamma)$ backgrounds.

- $65 \mathrm{GeV}<m(\ell \nu)<125 \mathrm{GeV}$. The neutrino momentum was calculated from momentum balance in the event. This requirement mostly suppresses the $W W \rightarrow \tau \nu_{\tau} j j$ background.

With these cuts, the selection efficiency is about $70 \%$, and the ratio of signal to background is approximately 20 .

Sensitivities to the $W W V$ couplings are calculated for the HISZ scenario [see Eqs. (61) - (63) ] from the results of a binned maximum log likelihood fit to event distributions, assuming an integrated luminosity of $500 \mathrm{pb}^{-1}$ which corresponds to several years of running. Figure 14a shows the $95 \%$ CL limit contours obtained at $176 \mathrm{GeV}$ and $190 \mathrm{GeV}$ from a fit to the $\cos \Theta_{W}, \cos \theta_{\ell}, \phi_{\ell}, \cos \theta_{j}$ and $\phi_{j}$ distributions, where the (down type) jet $j$ was chosen at random from the jet pair, i.e. it was assumed that quarks cannot be tagged. Close to the $W$ pair threshold, the gauge theory cancellations are not fully operative and the sensitivity to anomalous $W W V$ is limited. If the LEP II center of mass energy can be increased to $190 \mathrm{GeV}$, the sensitivity bounds improve by about a factor 1.5. The limits on $\Delta \kappa_{\gamma}^{0}$ and $\lambda_{\gamma}^{0}$ for $\sqrt{s}=176 \mathrm{GeV}$ and $190 \mathrm{GeV}$ in the HISZ scenario are summarized in Table 6 for the case where only one of the two couplings deviates from the SM at a time.

Note that the limits on $\Delta \kappa_{\gamma}^{0}$ and $\lambda_{\gamma}^{0}$ at LEP II are quite strongly correlated, in contrast to those obtained from $W \gamma$ and $W W, W Z$ production production in hadronic collisions. The much reduced correlations at hadron colliders are due to the high Tevatron and LHC center of mass energies, and the different high energy 
Figure 14: 95\% CL sensitivity limits from $e^{+} e^{-} \rightarrow W^{+} W^{-} \rightarrow \ell \nu j j$ at LEP II for an integrated luminosity of $500 \mathrm{pb}^{-1}$. a) Limit contours for $\sqrt{s}=176 \mathrm{GeV}$ and $190 \mathrm{GeV}$ from fitting all five angular distributions, assuming no quark tagging. b) Contours obtained assuming that no information about the hadronically decaying $W$ is used (dashed line), using all five angles assuming no quark tagging (solid line), and contours found for the hypothetical situation that all five angles are used and quarks are tagged with 100\% efficiency (dotted line).

Table 6: Expected 95\% CL limits on anomalous $W W V, V=\gamma, Z$, couplings from experiments at LEP II in the HISZ scenario [see Eqs. (61) - (63)] for two center of mass energies. The integrated luminosity assumed is $\int \mathcal{L} d t=500 \mathrm{pb}^{-1}$. Only one of the independent couplings is assumed to deviate from the SM at a time. The limits are obtained from a binned log likelihood fit to all five angles, assuming no quark tagging.

\begin{tabular}{|ccc|}
\hline dependent couplings & limit & limit \\
& $\sqrt{s}=176 \mathrm{GeV}$ & $\sqrt{s}=190 \mathrm{GeV}$ \\
\hline Eqs. (61) and (62) & $-0.19<\Delta \kappa_{\gamma}^{0}<0.21$ & $-0.13<\Delta \kappa_{\gamma}^{0}<0.14$ \\
$\lambda_{\gamma}=\lambda_{Z}$ & $-0.18<\lambda_{\gamma}^{0}<0.19$ & $-0.13<\lambda_{\gamma}^{0}<0.14$ \\
\hline
\end{tabular}


behavior of terms proportional to $\Delta \kappa_{\gamma}$ and $\lambda_{\gamma}$ in the helicity amplitudes. Figure 14b shows limit contours at $\sqrt{s}=176 \mathrm{GeV}$ for binning events in $\cos \Theta_{W}, \cos \theta_{\ell}$, and $\phi_{\ell}$ only (dashed line), all five angles assuming that quarks cannot be tagged (solid line), and for the hypothetical case where the quarks of the hadronically decaying $W$ boson are always tagged correctly (dotted line). The dotted line thus corresponds to the ultimate theoretical precision with which the anomalous couplings could be determined. Whereas the information obtained from the hadronically decaying $W$ does not affect the limits if only one of the two couplings is varied at a time, it reduces the correlations between $\Delta \kappa_{\gamma}^{0}$ and $\lambda_{\gamma}^{0}$ by approximately a factor 1.5 . Due to the relatively low center of mass energy, the limits which can be achieved at LEP II are very insensitive to the form factor scale and power assumed.

The contributions from $Z$ and photon exchange in $e^{+} e^{-} \rightarrow W^{+} W^{-}$tend to cancel. Therefore, if the $W W \gamma$ or $W W Z$ couplings only are allowed to deviate from the SM, somewhat more stringent limits are obtained than in the HISZ scenario used in our simulations.

Single photon production [32] at LEP II yields sensitivity limits on the $W W V$ couplings which are substantially weaker than those derived from $W$ pair production. The limits estimated from single $W$ production, on the other hand, are comparable to those obtained from $e^{+} e^{-} \rightarrow W^{+} W^{-}$83].

$Z \gamma V$ couplings can be probed in $Z \gamma$ production at LEP II. To illustrate the sensitivities which might be expected, 95\% CL limit contours for the $Z Z \gamma$ and $Z \gamma \gamma$ couplings were derived from $e^{+} e^{-} \rightarrow Z \gamma \rightarrow \bar{\nu} \nu \gamma$ and $e^{+} e^{-} \rightarrow Z \gamma \rightarrow \mu^{+} \mu^{-} \gamma$, respectively. For both processes a photon energy $E_{\gamma}>60 \mathrm{GeV}$, and $\left|\cos \theta_{\gamma}\right|<0.8$ was required. For single photon production, the cut on the photon energy significantly suppresses [32] the contribution from $t$-channel $W$ exchange to the $\bar{\nu}_{e} \nu_{e} \gamma$ final state, which is not included in the calculation used. The muon scattering angle, $\theta_{\mu}$, in $e^{+} e^{-} \rightarrow \mu^{+} \mu^{-} \gamma$ was required to satisfy $\left|\cos \theta_{\mu}\right|<0.927$ which corresponds to the L3 angular coverage for muons at LEP II. Muons are also required to have $p_{T}(\mu)>$ $10 \mathrm{GeV}$ and to be well isolated from the photon; $\Delta R(\mu, \gamma)>0.35$. In addition, a cut on the di-muon mass of $m(\mu \mu)>10 \mathrm{GeV}$ is imposed. A simplified model of the L3 detector is used to simulate detector effects.

Sensitivity bounds are calculated from a fit to the total cross section within cuts. The resulting $95 \%$ limit contours for a center of mass energy of $180 \mathrm{GeV}$, $\Lambda_{F F}=1 \mathrm{TeV}$, and an integrated luminosity of $500 \mathrm{pb}^{-1}$ are shown in Fig. 15. Since the LEP II center of mass energy will be significantly above the $Z \gamma$ threshold, the bounds derived on anomalous $Z \gamma V$ couplings vary only little within the expected range of center of mass energies expected $(\sqrt{s}=176 \mathrm{GeV}-190 \mathrm{GeV})$, in contrast to the situation encountered for $W$ pair production. The limits on $h_{30}^{V}$ and $h_{40}^{V}$ are summarized in Table 7 for the case where only one of the two couplings deviates from the SM at a time. For the $Z Z \gamma$ couplings, we also include the present L3 limits from $Z \rightarrow \bar{\nu} \nu \gamma$ for comparison. Due to the higher LEP II center of mass energy the present limits on $Z Z \gamma$ couplings from $Z \rightarrow \bar{\nu} \nu \gamma$ improve by a factor $1.6\left(h_{3}^{Z}\right)$ to $4.6\left(h_{4}^{Z}\right)$. The improvement is more pronounced for $h_{4}^{Z}$, due to the stronger growth with energy of the terms proportional to $h_{4}^{Z}$ in the helicity amplitudes. The limits 
Figure 15: 95\% CL sensitivity limits from $e^{+} e^{-} \rightarrow Z \gamma$ at LEP II for an integrated luminosity of $500 \mathrm{pb}^{-1}$. a) Limit contours for $Z Z \gamma$ couplings from single photon production. b) Sensitivity limits for $Z \gamma \gamma$ couplings from $e^{+} e^{-} \rightarrow \mu^{+} \mu^{-} \gamma$.

Table 7: Expected 95\% CL limits on anomalous $Z \gamma V V=\gamma, Z$, couplings from experiments at LEP II for $\sqrt{s}=180 \mathrm{GeV}$. The integrated luminosity assumed is $\int \mathcal{L} d t=500 \mathrm{pb}^{-1}$. Only one of the two couplings is assumed to deviate from the SM at a time. For comparison, we have also included the limits on $h_{30}^{Z}$ and $h_{40}^{Z}$ from $Z \rightarrow \bar{\nu} \nu \gamma$ at LEP [79]. The form factor scale chosen is $\Lambda_{F F}=1 \mathrm{TeV}$.

\begin{tabular}{|c|c|c|}
\hline reactions & \multicolumn{2}{|c|}{ limits } \\
\hline$e^{+} e^{-} \rightarrow Z \rightarrow \bar{\nu} \nu \gamma$ & $-0.79<h_{30}^{Z}<0.79$ & $-2.08<h_{40}^{Z}<2.08$ \\
\hline$e^{+} e^{-} \rightarrow Z \gamma \rightarrow \bar{\nu} \nu \gamma$ & $-0.50<h_{30}^{Z}<0.50$ & $-0.45<h_{40}^{Z}<0.45$ \\
\hline$e^{+} e^{-} \rightarrow Z \gamma \rightarrow \mu^{+} \mu^{-} \gamma$ & $-0.55<h_{30}^{\gamma}<0.55$ & $-0.48<h_{40}^{\gamma}<0.48$ \\
\hline
\end{tabular}


on $h_{30}^{V}$ and $h_{40}^{V}$ which can be achieved are quite similar at LEP II. The sensitivity bounds on $Z \gamma \gamma$ couplings are about $10 \%$ weaker than those found for $Z Z \gamma$ couplings. However, they are expected to significantly improve, if the angular distributions of the final state particles are analyzed instead of the total cross section.

\subsection{3 $W^{+} W^{-}$Production at the Next Linear Collider}

Since the LEP II center of mass energy is only slightly above the $W$ pair threshold, the SM gauge cancellations are not fully operative, and the sensitivity to anomalous gauge boson couplings is limited. Much better limits on $W W V$ and $Z \gamma V$ couplings will be possible at an $e^{+} e^{-}$collider operating in the several hundred $\mathrm{GeV}$ range or above. Such a machine will presumably be a linear collider. Current design studies for such a "Next Linear Collider" (NLC) foresee an initial stage with a center of mass energy of $500 \mathrm{GeV}$ and a luminosity of $8 \cdot 10^{33} \mathrm{~cm}^{-2} \mathrm{~s}^{-1}$. In a second stage, the energy is increased to $\sqrt{s}=1.5 \mathrm{TeV}$, with a luminosity of $1.9 \cdot 10^{34} \mathrm{~cm}^{-2} \mathrm{~s}^{-1} 84$.

As we have mentioned in Section 3.1, such a linear collider could also be operated as a $e \gamma, \gamma \gamma$ and $e^{-} e^{-}$collider, and a variety of processes can be used to constrain the vector boson self-interactions at the NLC. Since the limits obtained from $W$ pair production in the $e^{+} e^{-}$mode 85 are comparable or better than those obtained from other processes, we restrict ourselves to the process $e^{+} e^{-} \rightarrow W^{+} W^{-}$ in the following.

The extraction of limits on the $W W V$ couplings at the NLC [85, 86] follows the same strategy employed at LEP II. Again, only the $\ell \nu j j$ final state is analyzed. All five angles are used in the maximum likelihood fits. Two cuts are imposed. First, we require $\left|\cos \Theta_{W}\right|<0.8$. This ensures that the event is well within the detector volume. The second cut forces the $W^{+} W^{-}$invariant mass to be within a few $\mathrm{GeV}$ of the nominal $e^{+} e^{-}$center of mass energy, and ensures that the $W^{+}$and $W^{-}$invariant masses each are within a few $\mathrm{GeV}$ of the $W$ pole mass, $m_{W}$. In order to impose the second cut, we reconstruct the mass of the leptonically decaying $W$ $\left(m_{W_{1}}\right)$, and the mass of the hadronically decaying $W\left(m_{W_{2}}\right) \cdot m_{W_{2}}$ is reconstructed by imposing four energy momentum constraints and solving for the momentum vector of the neutrino from the leptonically decaying $W$, and $m_{W_{2}} \cdot m_{W_{1}}$ is then given by

$$
m_{W_{1}}=\left[\left(E_{\ell}+E_{\nu}\right)^{2}-\left(\mathbf{p}_{\ell}+\mathbf{p}_{\nu}\right)^{2}\right]^{1 / 2} .
$$

We then require

$$
\chi^{2}<2
$$

where $\chi^{2}$ is defined by

$$
\chi^{2}=\frac{\left(m_{W_{1}}-m_{W}\right)^{2}}{\Gamma_{W}^{2}}+\frac{\left(m_{W_{2}}-m_{W}\right)^{2}}{\Gamma_{W}^{2}} .
$$

Figure 16 shows the $95 \%$ CL contours for $\Delta \kappa_{\gamma}$ and $\lambda_{\gamma}$ at $\sqrt{s}=500 \mathrm{GeV}$ with $80 \mathrm{fb}^{-1}$, and at $\sqrt{s}=1.5 \mathrm{TeV}$ with $190 \mathrm{fb}^{-1}$ for the HISZ scenario [see Eqs. (61) 
Figure 16: The 95\% CL limit contours for $\Delta \kappa_{\gamma}$ and $\lambda_{\gamma}$ from $e^{+} e^{-} \rightarrow W^{+} W^{-}$at $\sqrt{s}=$ $500 \mathrm{GeV}$ with $80 \mathrm{fb}^{-1}$ (solid line), and at $\sqrt{\mathrm{s}}=1.5 \mathrm{TeV}$ with $190 \mathrm{fb}^{-1}$ (dashed line) for the HISZ scenario [see Eqs. 61) - 63)].

Table 8: Expected 95\% CL limits on anomalous $W W V, V=\gamma, Z$, couplings from experiments at the NLC in the HISZ scenario [see Eqs. (61) - 663)] for two center of mass energies and integrated luminosities. Only one of the independent couplings is assumed to deviate from the SM at a time. The limits are obtained from a log likelihood fit to all five angles, assuming no quark tagging.

\begin{tabular}{|ccc|}
\hline dependent couplings & limit & limit \\
& $\sqrt{s}=500 \mathrm{GeV}$ & $\sqrt{s}=1.5 \mathrm{TeV}$ \\
& $\int \mathcal{L} d t=80 \mathrm{fb}^{-1}$ & $\int \mathcal{L} d t=190 \mathrm{fb}^{-1}$ \\
\hline Eqs. (61) and (62) & $-0.0024<\Delta \kappa_{\gamma}<0.0024$ & $-5.2 \cdot 10^{-4}<\Delta \kappa_{\gamma}<5.2 \cdot 10^{-4}$ \\
$\lambda_{\gamma}=\lambda_{Z}$ & $-0.0018<\lambda_{\gamma}<0.0018$ & $-3.8 \cdot 10^{-4}<\lambda_{\gamma}<3.8 \cdot 10^{-4}$ \\
\hline
\end{tabular}


- (63)]. The limits for the case that only one of the two independent couplings deviates from the SM are summarized in Table 8. Depending on the energy and integrated luminosity of the NLC, the LEP II limits could be improved by two to three orders of magnitude. No form factor effects are taken into account in the bounds listed. However, due to the fixed center of mass energy, these effects can easily be incorporated. They result in a simple rescaling of the limits quoted.

The sensitivities for $Z_{\gamma} V$ couplings are expected to be of $\mathcal{O}\left(10^{-3}\right)$ at the NLC [87.

\section{Conclusions}

In this report, we have discussed the direct measurement of $W W V$ and $Z \gamma V$ couplings in present and future collider experiments. These couplings are defined through a phenomenological effective Lagrangian [see Eqs. (1) and (6)], analogously to the general vector and axial vector couplings, $g_{V}$ and $g_{A}$, for the coupling of gauge bosons to fermions. The major goal of such experiments will be the confirmation of the SM predictions. We have also reviewed our current theoretical understanding of anomalous gauge boson self-interactions. If the energy scale of the new physics responsible for the non-standard gauge boson couplings is $\sim 1 \mathrm{TeV}$, these anomalous couplings are expected to be no larger than $\mathcal{O}\left(10^{-2}\right)$.

Rigorously speaking, the three gauge boson vertices are unconstrained by current electroweak precision experiments. Such experiments only lead to bounds on the anomalous couplings if one assumes that cancellations between the coefficients of the effective Lagrangian of the underlying model are unnatural. Even in this case, the resulting bounds depend quite strongly on other parameters $\left(m_{H}, m_{t}\right)$, and anomalous couplings of $\mathcal{O}(1)$ are still allowed by current data (see Section 2.4).

Present data from di-boson production at the Tevatron and from single photon production at LEP yield bounds typically in the range of $0.5-3.0$. They are summarized in Tables 1 and $5 . \Delta \kappa_{\gamma}$ is currently constrained best by the process $p \bar{p} \rightarrow W^{+} W^{-}, W Z \rightarrow \ell \nu j j(\mathrm{CDF})$, whereas the best bound on $\lambda_{\gamma}$ originates from $W \gamma$ production at the Tevatron (DØ). The most precise limits on the $Z \gamma V$ couplings result from $e^{+} e^{-} \rightarrow \bar{\nu} \nu \gamma\left(\mathrm{L} 3 ; h_{3}^{Z}\right)$ and $Z \gamma$ production at the Tevatron (Dø; $\left.h_{4}^{V}, V=\gamma, Z\right)$. Although the present limits on $W W V$ and $Z \gamma V$ couplings are more than two orders of magnitude larger than what one expects from theoretical considerations if new physics exists at the $\mathrm{TeV}$ scale, these limits still provide valuable information on how well the vector boson self-interaction sector is tested experimentally at present.

Within the next 10 years, the limits on $W W V$ couplings are expected to improve by more than one order of magnitude by experiments conducted at the Tevatron and at LEP II. In Fig. 17 we compare the limits expected from $e^{+} e^{-} \rightarrow$ $W^{+} W^{-} \rightarrow \ell \nu j j, p \bar{p} \rightarrow W^{ \pm} \gamma \rightarrow e^{ \pm} \nu \gamma, p \bar{p} \rightarrow W^{ \pm} Z \rightarrow \ell_{1}^{ \pm} \nu_{1} \ell_{2}^{+} \ell_{2}^{-}$and $p \bar{p} \rightarrow W W, W Z \rightarrow$

$\ell \nu j j, \ell^{+} \ell^{-} j j$ in the HISZ scenario [see Eqs. (61) - (63)] for the envisioned energies and integrated luminosities. The limits expected from future Tevatron and LEP II experiments for $\Delta \kappa_{\gamma}$ are quite similar, whereas the Tevatron enjoys a clear advantage in constraining $\lambda_{\gamma}$, if correlations between the two couplings are taken into 
Figure 17: Comparison of the expected sensitivities on anomalous $W W V$ couplings in the HISZ scenario from $e^{+} e^{-} \rightarrow W^{+} W^{-} \rightarrow \ell \nu j j$ at LEP II and various processes at the Tevatron. 
account. It should be noted, however, that the strategies to extract information on vector boson self-interactions at the two machines are very different. At the Tevatron one exploits the strong increase of the anomalous contributions to the helicity amplitudes with energy to derive limits. At LEP II, on the other hand, information is extracted from the angular distributions of the final state fermions. Data from the Tevatron and LEP II thus yield complementary information on the nature of the $W W V$ couplings.

Because of the much higher energies accessible at the Tevatron and the steep increase of the anomalous contributions to the helicity amplitudes with energy, Tevatron experiments will be able to place significantly better limits (of $\mathcal{O}\left(10^{-2}-\right.$ $\left.10^{-3}\right)$ ) on the $Z \gamma V$ couplings than LEP II $(\approx 0.5)$. The Tevatron limits, however, do depend non-negligibly on the form factor scale assumed.

At the LHC one expects to probe anomalous $W W V$ couplings with a precision of $\mathcal{O}\left(10^{-1}-10^{-3}\right)$ (see Table 4 ) if the form factor scale $\Lambda_{F F}$ is larger than about $2 \mathrm{TeV}$. Therefore, it may be possible to probe anomalous $W W V$ couplings at the LHC at the level where one would hope to see deviations from the SM. The limits on the $Z \gamma V$ couplings are very sensitive to the value of $\Lambda_{F F}$. For $\Lambda_{F F} \geq 1.5 \mathrm{TeV}$, the bounds which can be achieved are of $\mathcal{O}\left(10^{-3}\right)$ for $h_{3}^{V}$, and of $\mathcal{O}\left(10^{-5}\right)$ for $h_{4}^{V}$. At the NLC, $W W V$ and $Z \gamma V$ couplings can be tested with a precision of $10^{-3}$ or better. Details depend quite sensitively on the center of mass energy and the integrated luminosity of the NLC. If new physics exists at the TeV scale, the NLC has the best chance to observe deviations from the SM through anomalous $W W V$ couplings.

\section{Acknowledgements}

We would like to thank our colleagues in CDF, DØ, and L3 for many valuable and stimulating discussions. We also thank Martin Einhorn for his critical comments. This work has been supported in part by the Department of Energy and by the N. S. E. R. C. of Canada and les Fonds F. C. A. R. du Québec.

\section{References}

1. D. Schaile, CERN-PPE/94-162 (preprint, October 1994), to appear in the Proceedings of the "27th International Conference on High Energy Physics", Glasgow, Scotland, July 1994; The LEP Collaborations, CERN-PPE/94-187 (preprint, November 1994).

2. F. Cuypers and K. Kolodziej, Phys. Lett. B344, 365 (1995); G. Abu Leil and W. J. Stirling, DTP-94-10 (preprint, June 1994); O. Eboli et al., Nucl. Phys. B411, 381 (1994); G. Belanger and F. Boudjema, Phys. Lett. B288, 201 (1992), and Phys. Lett. B288, 210 (1992).

3. K. Hagiwara, K. Hikasa, R. D. Peccei, D. Zeppenfeld, Nucl. Phys. B282, 253 (1987); K. Gaemers and G. Gounaris, Z. Phys. C1, 259 (1979).

4. H. Aronson, Phys. Rev. 186, 1434 (1969); K. J. Kim and Y.-S. Tsai, Phys. Rev. D7, 3710 (1973). 
5. S. Weinberg, Physica 96A, 327 (1979); J. Polchinski, Nucl. Phys. B231, 269 (1984); H. Georgi, Weak Interactions and Modern Particle Theory (Benjamin/Cummings Menlo Park, 1984); C. P. Burgess and J. A. Robinson, in BNL Summer Study on CP Violation, S. Dawson and A. Soni editors, (World Scientific, Singapore, 1991).

6. C. P. Burgess and D. London, Phys. Rev. Lett. 69, 3428 (1992), and Phys. Rev. D48, 4337 (1993).

7. W. Buchmüller and D. Wyler, Nucl. Phys. B268, 621 (1986).

8. K. Hagiwara, S. Ishihara, R. Szalapski, and D. Zeppenfeld, Phys. Lett. B283, 353 (1992), and Phys. Rev. D48, 2182 (1993).

9. B. Grinstein and M. B. Wise, Phys. Lett. B265, 326 (1991).

10. A. De Rújula, M. B. Gavela, P. Hernández, E. Massó, Nucl. Phys. B384, 3 (1992).

11. U. Baur and D. Zeppenfeld, Phys. Lett. B201, 383 (1988).

12. C. Arzt, M. B. Einhorn, and J. Wudka, Phys. Rev. D49, 1370 (1994); Nucl. Phys. B433, 41 (1994); M. B. Einhorn and J. Wudka, preprints NSF-ITP-9201 (1992) and UM-TH-92-25 (1992); J. Wudka, Int. J. Mod. Phys. A9, 2301 (1994).

13. S. Coleman, J. Wess, and B. Zumino, Phys. Rev. 177, 2239 (1969); E. C. Callan, S. Coleman, J. Wess, and B. Zumino, Phys. Rev. 177, 2247 (1969); J. Gasser and H. Leutwyler, Ann. Phys. (NY) 158, 142 (1984); M. S. Chanowitz, M. Golden, and H. Georgi, Phys. Rev. D36, 1490 (1987).

14. A. Manohar and H. Georgi, Nucl. Phys. B234, 189 (1984); H. Georgi and L. Randall, Nucl. Phys. B276, 241 (1986); H. Georgi, Phys. Lett. B298, 187 (1993).

15. T. Appelquist and C. Bernard, Phys. Rev. D22, 200 (1980); A. Longhitano, Nucl. Phys. B188, 118 (1981); T. Appelquist and G.-H. Wu, Phys. Rev. D48, 3235 (1993).

16. S. Dawson and G. Valencia, BNL-60949 (preprint, October 1994), to appear in Nucl. Phys. B.

17. S. Dawson and G. Valencia, Phys. Rev. D49, 2188 (1994); Phys. Lett. B333, 207 (1994), and erratum to appear.

18. J. M. Cornwall, D. N. Levin, and G. Tiktopoulos, Phys. Rev. Lett. 30, 1268 (1973), Phys. Rev. D10, 1145 (1974); C. H. Llewellyn Smith, Phys. Lett. B46, 233 (1973); S. D. Joglekar, Ann. Phys. 83, 427 (1974).

19. U. Baur and E. L. Berger, Phys. Rev. D47, 4889 (1993).

20. S.J. Brodsky and J.D. Sullivan, Phys. Rev. 156, 1644 (1967); F. Herzog, Phys. Lett. B148, 355 (1984); (E)B155, 468 (1985); A. Grau and J.A. Grifols, Phys. Lett. B154, 283 (1985); J.C. Wallet, Phys. Rev. D32, 813 (1985); P. Méry, S.E. Moubarik, M. Perrottet, and F.M. Renard, Z. Phys. C46, 229 (1990); 
F. Boudjema, K. Hagiwara, C. Hamzaoui, and K. Numata, Phys. Rev. D43, 2223 (1991).

21. S. P. Chia, Phys. Lett. B240, 465 (1990); K. Numata, Z. Phys. C52, 691 (1991); K. A. Peterson, Phys. Lett. B282, 207 (1992); T. G. Rizzo, Phys. Lett. B315, 471 (1993); U. Baur, Proceedings of the "Workshop on B Physics at Hadron Accelerators", Snowmass, Colorado, June 1993, p. 455; X. He and B. McKellar, Phys. Lett. B320, 165 (1994). R. Martinez, M. A. Pérez, and J. J. Toscano, Phys. Lett. B340, 91 (1994).

22. M. S. Alam et al. (CLEO Collaboration), CLNS-94-1314 (preprint, December 1994).

23. G. Baillie, Z. Phys. C61, 667 (1994).

24. O. Eboli et al., Phys. Lett. B339, 119 (1994); F. M. Renard and C. Verzegnassi, Phys. Lett. B345, 500 (1995).

25. K. Hagiwara, S. Matsumoto, D. Haidt, and C. S. Kim, Z. Phys. C64, 559 (1994); S. Matsumoto, KEK-TH-418 (preprint, November 1994).

26. M. E. Peskin and T. Takeuchi, Phys. Rev. Lett. 65, 964 (1990).

27. See also I. Maksymyk, C. P. Burgess, and D. London, Phys. Rev. D50, 529 (1994).

28. C. P. Burgess, S. Godfrey, H. König, D London, and I. Maksymyk, Phys. Rev. D49, 6115 (1994).

29. P. Hernández and F. J. Vegas, Phys. Lett. B307, 116 (1993); D. Choudhury, P. Roy, and R. Sinha, TIFR-TH/93-08 (1993) (unpublished).

30. R. W. Brown et al., Phys. Rev. D20, 1164 (1979); K. O. Mikaelian et al., Phys. Rev. Lett. 43, 746 (1979).

31. U. Baur, T. Han, and J. Ohnemus, Phys. Rev. Lett. 72, 3941 (1994).

32. G. Couture and S. Godfrey, Phys. Rev. D50, 5607 (1994).

33. V. Barger and T. Han, Phys. Lett. B241, 127 (1990); G. Couture and S. Godfrey, Phys. Rev. D49, 5709, (1994).

34. I. F. Ginzburg et al., Nucl. Instr. and Meth. 205, 47 (1983), and Nucl. Instr. and Meth. 219, 5 (1984); V. I. Telnov, Nucl. Instr. and Meth. A294, 72 (1990).

35. E. Yehudai, Phys. Rev. D41, 33 (1990).

36. S. Y. Choi and F. Schrempp, Phys. Lett. B272, 149 (1991).

37. E. Yehudai, Phys. Rev. D44, 3434 (1991).

38. S. Y. Choi, KEK-TH-420, (preprint, December 1994).

39. D. Choudhury and F. Cuypers, Phys. Lett. B325, 500 (1994), and Nucl. Phys. B429, 33 (1994).

40. A. Miyamoto, Proceedings of the "Workshop on Physics and Experiments with Linear $e^{+} e^{-}$Colliders", Waikoloa, Hawaii, April 1993, p. 141. 
41. U. Baur and D. Zeppenfeld, Nucl. Phys. B325, 253 (1989); U. Baur et al., Proceedings of the "ECFA Large Hadron Collider Workshop", Aachen, Germany, October 1990, Vol. II, p. 956; C. S. Kim, J. Lee, and H. S. Song, Z. Phys. C63, 673 (1994).

42. U. Baur and E. L. Berger, Phys. Rev. D41, 1476 (1990).

43. S. Willenbrock and D. Zeppenfeld, Phys. Rev. D37, 1775 (1988).

44. F. Abe et al. (CDF Collaboration), Phys. Rev. Lett. 74, 1936 (1995).

45. J. Ellison (DØ Collaboration), FERMILAB-Conf-94/329-E (preprint, November 1994), to appear in the Proceedings of the "DPF'94 Conference", Albuquerque, NM, August 1994; H. Aihara, talk given at the "International Symposium on Vector Boson Self-interactions", UCLA, February 1995.

46. F. Abe et al. (CDF Collaboration), Phys. Rev. Lett. 74, 1941 (1995).

47. S. Abachi et al. (DØ Collaboration), FERMILAB-Pub-95/042-E, (preprint, March 1995), submitted to Phys. Rev. Lett.

48. T. A. Fuess (CDF Collaboration), FERMILAB-Conf-94/283-E, (preprint, September 1994), to appear in the Proceedings of the "DPF'94 Conference", Albuquerque, NM, August 1994; F. Abe et al. (CDF Collaboration), FERMILAB-Conf-94/158-E (preprint, June 1994), contributed paper to the "27th International Conference on High Energy Physics", Glasgow, Scotland, July 20 - 27, 1994; F. Abe et al. (CDF Collaboration), FERMILAB-Pub95/036-E (preprint, March 1995), submitted to Phys. Rev. Lett.

49. S. Abachi et al. (DØ Collaboration), FERMILAB-Pub-95/044-E, (preprint, March 1995), submitted to Phys. Rev. Lett.

50. F. Abe et al. (CDF Collaboration), FERMILAB-Pub-94/244-E (preprint, July 1994), to appear in Phys. Rev. D.

51. S. Errede, FERMILAB-Conf-94/306-E (preprint, September 1994), to appear in the Proceedings of the "27th International Conference on High Energy Physics", Glasgow, Scotland, July 1994.

52. J. Ohnemus, Phys. Rev. D44, 1403 (1991); Phys. Rev. D44, 3477 (1991); Phys. Rev. D50, 1931 (1994); J. Ohnemus and J. Owens, Phys. Rev. D43, 3626 (1991); S. Frixione, Nucl. Phys. B410, 280 (1993); S. Frixione, P. Nason, and S. Ridolfi, Nucl. Phys. B383, 3 (1992).

53. A. Martin, R. G. Roberts, and W. J. Stirling, Phys. Lett. B306, 145 (1993).

54. F. Abe et al. (CDF Collaboration), Phys. Rev. Lett. 74, 850 (1995).

55. K. Hagiwara, J. Woodside, and D. Zeppenfeld, Phys. Rev. D41, 2113 (1990).

56. T. Han, R. Meng, and J. Ohnemus, Nucl. Phys. B384, 59 (1992).

57. J. Alitti et al. (UA2 Collaboration), Phys. Lett. B277, 194 (1992).

58. D. Amidei et al., FERMILAB-Conf-94/249-E (preprint, August 1994), to appear in the Proceedings of the "DPF'94 Conference", Albuquerque, NM, August 1994; S. Holmes and V. A. Yarba, FERMILAB-Conf-94/360 (preprint, Octo- 
ber 1994), presented at the "XIV Conference on Charged Particle Accelerators", Protvino, Russia, October 1994.

59. U. Baur, J. Ohnemus, and T. Han, Phys. Rev. D51, 3381 (1995).

60. F. A. Berends et al., Nucl. Phys. B357, 32 (1991).

61. U. Baur and D. Zeppenfeld, Nucl. Phys. B308, 127 (1988).

62. The LHC Study Group, Design Study of the Large Hadron Collider, CERN 91-03, (1991).

63. D. Gingrich et al. (ATLAS Collaboration), ATLAS Letter of Intent, CERNLHCC-92-4 (October 1992); W. W. Armstrong et al. (ATLAS Collaboration), ATLAS Technical Design Report, CERN-LHCC-94-43 (December 1994).

64. M. Della Negra et al. (CMS Collaboration), CMS Letter of Intent, CERNLHCC-92-3 (October 1992); G. L. Bayatian et al. (CMS Collaboration), CMS Technical Design Report, CERN-LHCC-94-38 (December 1994).

65. U. Baur, T. Han, and J. Ohnemus, Phys. Rev. D48, 5140 (1993); J. Ohnemus, Phys. Rev. D51, 1068 (1995).

66. E. Maina and S. Moretti, Phys. Lett. B286, 370 (1992).

67. U. Baur and A. Stange, Proceedings of the "Workshop on Physics at Current Accelerators and the Supercollider", Argonne, June 1993, p. 319.

68. C. Albajar et al., Proceedings of the "ECFA Large Hadron Collider Workshop", Aachen, Germany, 1990, Vol. II, p. 621.

69. E. Reya et al., Proceedings of the "ECFA Large Hadron Collider Workshop", Aachen, Germany, October 1990, Vol. II, p. 296.

70. D. Zhu, Phys. Rev. D22, 2266 (1980); C. J. Goebel et al., Phys. Rev. D23, 2682 (1981).

71. S. J. Brodsky and R. W. Brown, Phys. Rev. Lett. 49, 966 (1982); R. W. Brown et al., Phys. Rev. D28, 624 (1983); R. W. Brown and K. L. Kowalski, Phys. Rev. D29, 2100 (1984).

72. J. Smith, D. Thomas, and W. L. van Neerven, Z. Phys. C44, 267 (1989); S. Mendoza, J. Smith, and W. L. van Neerven, Phys. Rev. D47, 3913 (1993).

73. G. Valenzuela and J. Smith, Phys. Rev. D31, 2787 (1985).

74. J. Gunion, Z. Kunszt, and M. Soldate, Phys. Lett. B163, 389 (1985); J. Gunion and M. Soldate, Phys. Rev. D34, 826 (1986); W. J. Stirling et al., Phys. Lett. B163, 261 (1985).

75. U. Baur, S. Errede, and G. Landsberg, Phys. Rev. D50, 1917 (1994).

76. C. Bilchak, R. Brown, and J. Stroughair, Phys. Rev. D29, 375 (1984).

77. S. Frixione, P. Nason, and S. Ridolfi, Nucl. Phys. B383, 3 (1992).

78. U. Baur, S. Errede, and J. Ohnemus, Phys. Rev. D48, 4103 (1993).

79. M. Acciarri et al. (L3 Collaboration), Phys. Lett. B346, 190 (1995).

80. R. L. Sekulin, Phys. Lett. B338, 369 (1994). 
81. M. Bilenky et al., Nucl. Phys. B409, 22 (1993); M. Diehl and O. Nachtmann, Z. Phys. C62, 397 (1994); C. G. Papadopoulos, DTP/95/20 (preprint, March 1995).

82. F. Renard, Nucl. Phys. B196, 93 (1981); P. Méry, M. Perrottet, and F. Renard, Z. Phys. C38, 579 (1987); A. Barroso and L. Bento, Phys. Rev. D38, 2742 (1988).

83. E. N. Argyres and C. G. Papadopoulos, Phys. Lett. B263, 298 (1991); C. G. Papadopoulos, Phys. Lett. B333, 202 (1994).

84. R. D. Ruth, SLAC-PUB-6751 (preprint, March 1995), to appear in "Report of the DPF Committee for Long Term Planning".

85. P. Mättig et al., Proceedings of the Workshop " $e^{+} e^{-}$Collisions at $500 \mathrm{GeV}$ : The Physics Potential, Munich, Annecy, Hamburg, 1991, Vol. A, p. 223; M. Bilenky et al., Nucl. Phys. B419, 240 (1994); T. Barklow, Proceedings of the Workshop "Physics and Experiments with Linear Colliders", Saariselka, Finland, 1991, Vol. I, p. 423.

86. T. Barklow, SLAC-PUB-6618 (preprint, August 1994), to appear in the Proceedings of the "DPF'94 Conference", Albuquerque, NM, August 1994.

87. F. Boudjema, Proceedings of the Workshop " $e^{+} e^{-}$Collisions at $500 \mathrm{GeV}$ : The Physics Potential, Munich, Annecy, Hamburg, 1991, Vol. B, p. 757. 